\title{
PROBLEMS IN NUMBER THEORY FROM BUSY BEAVER COMPETITION
}

\author{
PASCAL MICHEL
}

Équipe de Logique Mathématique, Institut de Mathématiques de Jussieu-Paris Rive Gauche, UMR 7586, Bâtiment Sophie Germain, case 7012, 75205 Paris Cedex 13, France and Université de CergyPontoise, IUFM, F-95000 Cergy-Pontoise, France

e-mail address: michel@math.univ-paris-diderot.fr

\begin{abstract}
By introducing the busy beaver competition of Turing machines, in 1962, Rado defined noncomputable functions on positive integers. The study of these functions and variants leads to many mathematical challenges. This article takes up the following one: How can a small Turing machine manage to produce very big numbers? It provides the following answer: mostly by simulating Collatz-like functions, that are generalizations of the famous $3 x+1$ function. These functions, like the $3 x+1$ function, lead to new unsolved problems in number theory.
\end{abstract}

\section{INTRODUCTION}

1.1. A well defined noncomputable function. It is easy to define a noncomputable function on nonnegative integers. Indeed, given a programming language, you produce a systematic list of the programs for functions, and, by diagonalization, you define a function whose output, on input $n$, is different from the output of the $n$th program. This simple definition raises many problems: Which programming language? How to list the programs? How to choose the output?

In 1962, Rado [Ra62] gave a practical solution by defining the busy beaver game, also called now the busy beaver competition. Consider all Turing machines on one infinite tape, with $n$ states (plus a special halting state), and two symbols (1, and the blank symbol 0 ), and launch all of them on a blank tape. Define $S(n)$ as the maximum number of computation steps made by such a machine before it stops, and define $\Sigma(n)$ as the maximum number of symbols 1 left on the tape by a machine when it stops. Then functions $S$ and $\Sigma$ are noncomputable, and, moreover, grow faster than any computable function, that is, for any computable function $f$, there exists an integer $N$ such that, for any $n \geq N$, $S(n)>\Sigma(n)>f(n)$.

2012 ACM CCS: [Theory of computation]: Models of computation-Computability-Turing machines.

Key words and phrases: busy beaver, Collatz-like functions.

Corresponding address: 59 rue du Cardinal Lemoine, 75005 Paris, France. 
More than fifty years later, no better choice has been found for a practical noncomputable function. Only variants of Rado's definition have been proposed. So, in 1988, Brady [Br88] defined similar functions $S(n, m)$ and $\Sigma(n, m)$ for $n \times m$ Turing machines, that is Turing machines with $n$ states and $m$ symbols. He also introduced analogous functions for two-dimensional Turing machines and "turNing machines", later resumed and expanded by Tim Hutton [HuW]. Bátfai [Ba09a, Ba09b] relaxed the rule about head moving, by allowing the head to stand still.

In this article, we will consider functions $S(n, m)$ and $\Sigma(n, m)$. Recall that $S(n)=$ $S(n, 2)$ and $\Sigma(n)=\Sigma(n, 2)$.

1.2. Computing the values of noncomputable functions. The busy beaver functions $S$ and $\Sigma$ are explicitely defined, and it is possible to compute $S(n, m)$ and $\Sigma(n, m)$ for small $n$ and $m$. In the first article on busy beavers, Rado $[\operatorname{Ra62}$ gave $\Sigma(2)=2$ and $\Sigma(3) \geq 6$. These results show that two problems are at stake:

- Problem 1: To give lower bounds on $S(n, m)$ and $\Sigma(n, m)$ by finding Turing machines with high scores.

- Problem 2: To compute $S(n, m)$ and $\Sigma(n, m)$ by proving that no Turing machines do better than the known best ones.

Problem 1 can be tackled either by hand search, as did, for example, Green [Gr64] and Lynn [Ly72, or by computer search, using acceleration techniques of computation and, for example, simulated annealing, as did T. and S. Ligocki [Li05].

Solving Problem 2 requires more work to be done: clever enumeration of $n \times m$ Turing machines, simulation of computation with acceleration techniques, proofs of non-halting for the machines that do not halt.

Currently, the following results are known (see Michel Mi09, MiWa] for a historical survey):

- $S(2)=6$ and $\Sigma(2)=4$ (Rado Ra62]),

- $S(3)=21$ and $\Sigma(3)=6$ (Lin and Rado LR65),

- $S(4)=107$ and $\Sigma(4)=13$ (Brady Br66, Br83, Machlin and Stout MS90]),

- $S(5) \geq 47,176,870$ and $\Sigma(5) \geq 4098$ (Marxen and Buntrock [MB90]),

- $S(6)>7.4 \times 10^{36534}$ and $\Sigma(6)>3.5 \times 10^{18267}(\mathrm{P}$. Kropitz in 2010),

- $S(2,3)=38$ and $\Sigma(2,3)=9$ (Lafitte and Papazian [LP07]),

- $S(3,3)>1.1 \times 10^{17}$ and $\Sigma(3,3) \geq 347,676,383$ (T. and S. Ligocki in 2007),

- $S(4,3)>1.0 \times 10^{14072}$ and $\Sigma(4,3)>1.3 \times 10^{7036}$ (T. and S. Ligocki in 2008),

- $S(2,4) \geq 3,932,964$ and $\Sigma(2,4) \geq 2050$ (T. and S. Ligocki in 2005),

- $S(3,4)>5.2 \times 10^{13036}$ and $\Sigma(3,4)>3.7 \times 10^{6518}$ (T. and S. Ligocki in 2007),

- $S(2,5)>1.9 \times 10^{704}$ and $\Sigma(2,5)>1.7 \times 10^{352}$ (T. and S. Ligocki in 2007),

- $S(2,6)>2.4 \times 10^{9866}$ and $\Sigma(2,6)>1.9 \times 10^{4933}$ (T. and S. Ligocki in 2008).

In order to achieve these results, many computational and mathematical challenges had to be taken up.

A: Computational challenges.

A1. To generate all $n \times m$ Turing machines, or rather, to treat all cases without having to generate all $n \times m$ Turing machines.

A2. To simulate the computation of a machine by using acceleration techniques (see Marxen and Buntrock MB90, Marxen [MaW]). 
A3. To gave automatic proofs that non-halting machines do not halt (see Brady Br83], Marxen and Buntrock [MB90, Machlin and Stout [MS90, Hertel [He09, Lafitte and Papazian [LP07]).

B: Mathematical challenges.

B1. To prove by hand that a non-halting machine that resists the computational proof does not halt.

B2. To understand how the Turing machines that reach high scores manage to do it.

1.3. Facing open problems in number theory. Let us come back to mathematical challenge $B 1$. For example, the computational study of $5 \times 2$ Turing machines by Marxen and Buntrock [MB90, Skelet [GeW] and Hertel [He09] left holdouts that needed to be analyzed by hand. Marxen and Buntrock [MB90] gave an unsettled $5 \times 2$ Turing machine, named \#4, that turned out to never halt, by an intricate analysis.

Actually, the halting problem for Turing machines launched on a blank tape is $\mathrm{m}$ complete, and this implies that this problem is as hard as the problem of the provability of a mathematical statement in a logical theory such as ZFC (Zermelo Fraenkel set theory with axiom of choice). So, when Turing machines with more and more states and symbols are studied, potentially all theorems of mathematics will be met. When more and more non-halting Turing machines are studied to be proved non-halting, one has to expect to face hard open problems in mathematics, that is problems that current mathematical knowledge can't settle.

Consider now mathematical challenge B2, which is the very subject of this article.

From 1983 to 1989 , several $5 \times 2$ Turing machines with high scores were discovered by Uwe Schult, by George Uhing, and by Heiner Marxen and Jürgen Buntrock. Michel Mi92, Mi93 analyzed some of these machines and found that their behavior is Collatzlike, which implies that the halting problems on general inputs for these machines are open problems in number theory (see Table 1).

From 2005 and 2007, many $3 \times 3,2 \times 4$ and $2 \times 5$ Turing machines with high scores were discovered, mainly by two teams: the French one of Grégory Lafitte and Christophe Papazian, and the father-and-son collaboration of Terry and Shawn Ligocki. Collatz-like behavior of these champions seems to be the rule (see Tables 3 , 4 and 5).

However, the behaviors of $6 \times 2$ Turing machines display some variety. Many machines were discovered, from 1990 to 2010, by Heiner Marxen and Jürgen Buntrock, by Terry and Shawn Ligocki and by Pavel Kropitz. The analyses of some of these machines, by Robert Munafo, Clive Tooth, Shawn Ligocki and the author, show that the behaviors can be Collatz-like, exponential Collatz-like, loosely Collatz-like, or definitely not Collatz-like. Almost all of them raise open problems (see Table 2).

Note: The Turing machines listed in Tables 15 are those for which an analysis is known by the author. The machines without references for the study of behavior were analyzed by the author [Mi09, MiWb]. Many other machines are waiting for their analyses.

1.4. Collatz functions, Collatz-like functions and other functions. The $3 x+1$ function, or Collatz function, is the function $T$ on positive integers defined by

$$
T(n)=\left\{\begin{array}{cl}
n / 2 & \text { if } n \text { is even } \\
(3 n+1) / 2 & \text { if } n \text { is odd }
\end{array}\right.
$$


This function is famous because, when it is iterated on a positive integer, it seems to lead to the loop $2,1,2,1, \ldots$ Is it always true? This is an open problem. See Lagarias La85, La03, La06, La10] for more information.

It is natural to generalize the definition of the $3 x+1$ function by replacing $n$ even, $n$ odd by $n \equiv 0, \ldots, d-1(\bmod d)$, and by replacing $n / 2,(3 n+1) / 2$ by $a n+b$ for rational numbers $a, b$. Unfortunately, no name for such functions is currently taken for granted. Formal definitions were given by Rawsthorne [Ra85, who proposed Collatz-type iteration functions, by Buttsworth and Matthews [BM90, who proposed generalized Collatz mappings, by Kaščák [Ka92, who proposed one-state linear operator algorithms (OLOA), and by Kohl [Ko07], who proposed residue-class-wise affine functions (RCWA). Without giving a formal definition, Lagarias La85 proposed periodically linear functions, and Wagon Wa85 proposed Collatz-like functions.

We will choose the following definitions.

Definition 1.1. A mapping $f: \mathbb{Z} \rightarrow \mathbb{Z}$ is a generalized Collatz mapping if there exists an integer $d \geq 2$ such that the following three equivalent conditions are satisfied:

(i) (see Wi98, p.14]) There exist rational numbers $q_{0}, \ldots, q_{d-1}, r_{0}, \ldots, r_{d-1}$, such that, for all $i, 0 \leq i \leq d-1$, we have $q_{i} d \in \mathbb{Z}, q_{i} i+r_{i} \in \mathbb{Z}$, and, for all $n \in \mathbb{Z}, f(n)=q_{i} n+r_{i}$ if $n \equiv i(\bmod d)$.

(ii) (see BM90]) There exist integers $m_{0}, \ldots, m_{d-1}, p_{0}, \ldots, p_{d-1}$, such that, for all $i$, $0 \leq i \leq d-1$, we have $p_{i} \equiv i m_{i}(\bmod d)$ and, for all $n \in \mathbb{Z}, f(n)=\left(m_{i} n-p_{i}\right) / d$ if $n \equiv i(\bmod d)$.

(iii) There exist integers $a_{0}, \ldots, a_{d-1}, b_{0}, \ldots, b_{d-1}$, such that we have, for all $i, 0 \leq i \leq d-1$, for all $n \in \mathbb{Z}, f(d n+i)=a_{i} n+b_{i}$.

These definitions are easily seen to be equivalent: we have $a_{i}=m_{i}=q_{i} d$ and $b_{i}=\left(i m_{i}-\right.$ $\left.p_{i}\right) / d=q_{i} i+r_{i}$.

The definitions above concern total functions, but, in this article, we always deal with partial functions and functions with parameters, so we introduce the following definitions.

Definition 1.2. A partial function $f: \mathbb{Z} \rightarrow \mathbb{Z}$ is a generalized Collatz function, or a pure Collatz-like function (without parameter) if, in the previous definition, $f(d n+i)$ can be undefined for one or many $i, 0 \leq i \leq d-1$.

Definition 1.3. A partial function $f: \mathbb{Z} \times \mathbb{Z} \rightarrow \mathbb{Z} \times \mathbb{Z}$ is a pure Collatz-like function with parameter if there exist an integer $d \geq 2$, integers $a_{0}, \ldots, a_{d-1}, b_{0}, \ldots, b_{d-1}$, a set $S$ of integers and a function $p:\{0, \ldots, d-1\} \times S \rightarrow S$ such that, for all $i, 0 \leq i \leq d-1$, for all $n \in \mathbb{Z}$, for all $s \in S, f(d n+i, s)=\left(a_{i} n+b_{i}, p(i, s)\right)$ or is undefined.

Definition 1.4. If, in the definitions above, $a_{i}=a$ for all $i, 0 \leq i \leq d-1$, we say that $f$ is pure Collatz-like of type $d \rightarrow a$.

We also need to define a new type of function, as follows.

Definition 1.5. A partial function $f: \mathbb{Z} \rightarrow \mathbb{Z}$ is an exponential Collatz-like function if there exist integers $d, p \geq 2$, integers $a_{0}, \ldots, a_{d-1}, b_{0}, \ldots, b_{d-1}, c_{0}, \ldots, c_{d-1}$, such that, for all $i, 0 \leq i \leq d-1$, all $n \in \mathbb{Z}, f(d n+i)=\left(a_{i} p^{n}+b_{i}\right) / c_{i}$ or is undefined. In this definition, integers $p, a_{i}, b_{i}, c_{i}$ are chosen such that $\left(a_{i} p^{n}+b_{i}\right) / c_{i}$ is an integer for all $n \in \mathbb{Z}$.

Currently, no study of this type of function is known. Note that iterates $f(n), f^{2}(n), \ldots$ grow much faster for exponential Collatz-like functions than for pure Collatz-like functions. 
1.5. From Collatz-like functions to high scores. The Turing machines studied in this article have behaviors modeled on iterations of functions, where halting configurations correspond to undefined values of functions.

How do Turing machines simulate Collatz-like functions?

First note that Baiocchi [Ba98, Margenstern [Ma00] and Michel [Mi14 found Turing machines that simulate the $3 x+1$ function with a very small number of states and symbols. In these articles, clever tricks were designed to minimize the size of the Turing machines.

On the other hand, the Turing machines in the present article were frontally attacked, and it is with handsight that they were found to simulate Collatz-like functions.

Note also that, while the theorems giving the behavior of Turing machines below are hard to be formally proven, they can be easily verified for the small values of the parameters, by using programs simulating the Turing machines.

In Section 3, we present a $3 \times 3$ Turing machine $M_{1}$ whose behavior is pure Collatz-like, of type $8 \rightarrow 14$. In Section 4 , we present a $2 \times 4$ Turing machine $M_{2}$ whose behavior is pure Collatz-like with parameter, of type $3 \rightarrow 5$. In Section 5, we present a $2 \times 5$ Turing machine $M_{3}$ whose behavior is pure Collatz-like with parameter, of type $2 \rightarrow 3$. Thus, the halting problem for machines $M_{1}, M_{2}$ and $M_{3}$ depends on an open problem about iterating Collatz-like functions.

In Section 6, we present a $6 \times 2$ Turing machine $M_{4}$ whose behavior is exponential Collatz-like.

In Section 7, we present a $6 \times 2$ Turing machine $M_{5}$ whose behavior depends on iterating a partial function $g_{5}(n, p)$. Without being Collatz-like, this function seems to share some properties with Collatz-like functions.

In Section 8, we present a $6 \times 2$ Turing machine $M_{6}$ whose behavior looks like a loosely Collatz-like behavior with parameter, of type $2 \rightarrow 5$. The novelty is that a potentially infinite set of rules seems to be necessary to completely describe the behavior of the machine on inputs $00 x, x \in\{0,1\}^{*}$. A string $x \in\{0,1\}^{*}$ ending with symbol 1 can be taken as the binary representation of a number $p$, read in the opposite direction, so $x=\mathrm{R}(\operatorname{bin}(p))$, where $\operatorname{bin}(p)$ is the usual binary representation of $p$, and $\mathrm{R}(w)$ is the reverse of string $w$, that is $\mathrm{R}\left(w_{1} \ldots w_{n}\right)=w_{n} \ldots w_{1}$. In Table 2 we write " $\mathrm{R}(\operatorname{bin}(p))$ " to indicate the machines with a behavior involving a potentially infinite set of rules. Of course, only a finite subset of these rules are used when the machine is launched on a blank tape.

In Section 9, we present a $6 \times 2$ Turing machine $M_{7}$ whose behavior on the blank tape depends on configurations $C(n)$ all of them provably leading to a halting configuration. We present such a machine to show how a Turing machine can take a long time to stop without calling for Collatz-like functions.

\section{Preliminaries}

A Turing machine involved in the busy beaver competition is defined as follows. It has a tape made of cells, infinite in both directions. Each cell contains a symbol, and one head can move on the tape and read and write a symbol on a cell. The Turing machine can be in a finite number of states. A computation of the Turing machine is a sequence of steps. In a step of computation, according to the current state and the symbol read by the head on the current cell, the head writes a symbol on the cell, moves to the next cell on the right side or on the left side, and the machine enters a new state. 


\begin{tabular}{|c|c|c|}
\hline Machine & Behavior & Study of behavior \\
\hline $\begin{array}{l}\text { January } 1983 \\
\text { Uwe Schult } \\
\quad \sigma=501 \\
s=134,467\end{array}$ & $\begin{array}{c}\text { Pure Collatz-like }(4 \rightarrow 9) \\
\text { without parameter } \\
7 \text { rules } \\
7 \text { transitions }\end{array}$ & $\begin{array}{l}\text { Robinson, } \\
\text { cited in De84. } \\
\text { Michel } \text { Mi92. }\end{array}$ \\
\hline $\begin{array}{c}\text { December } 1984 \\
\text { George Uhing } \\
\quad \sigma=1915 \\
s=2,133,492\end{array}$ & $\begin{array}{c}\text { Pure Collatz-like }(3 \rightarrow 8) \\
\text { with parameter } \\
5 \text { rules } \\
9 \text { transitions }\end{array}$ & Michel Mi92 \\
\hline $\begin{array}{l}\text { February } 1986 \\
\text { George Uhing } \\
\quad \begin{array}{c}\sigma=1471 \\
s=2,358,064\end{array}\end{array}$ & $\begin{array}{c}\text { Pure Collatz-like }(8 \rightarrow 15) \\
\text { without parameter } \\
5 \text { rules } \\
11 \text { transitions }\end{array}$ & \\
\hline $\begin{array}{c}\text { August } 1989 \\
\text { Marxen, Buntrock } \\
\sigma=4098 \\
s=11,798,826\end{array}$ & $\begin{array}{c}\text { Pure Collatz-like }(3 \rightarrow 5) \\
\text { without parameter } \\
3 \text { rules } \\
15 \text { transitions }\end{array}$ & Michel [Mi92] \\
\hline $\begin{array}{c}\text { September } 1989 \\
\text { Marxen, Buntrock } \\
\sigma=4097 \\
s=23,554,764\end{array}$ & $\begin{array}{c}\text { Pure Collatz-like }(3 \rightarrow 5) \\
\text { without parameter } \\
3 \text { rules } \\
15 \text { transitions }\end{array}$ & Michel Mi92 \\
\hline $\begin{array}{c}\text { September } 1989 \\
\text { Marxen, Buntrock } \\
\sigma=4098 \\
s=47,176,870\end{array}$ & $\begin{array}{c}\text { Pure Collatz-like }(3 \rightarrow 5) \\
\text { without parameter } \\
3 \text { rules } \\
15 \text { transitions }\end{array}$ & Michel Mi93 \\
\hline
\end{tabular}

Table 1: Study of behavior of $5 \times 2$ machines. For each machine, in the first column, one can find when it was discovered, by whom, the number $\sigma$ of non-blank symbols left on the tape when the machine halts, and the number $s$ of steps of the computation. In the second column, the behavior of the machine is given, and we refer to Def. 1.2-1.4 for the precise definition of pure Collatz-like function, with and without parameter, of type $d \rightarrow a$. The number of rules gives roughly the length of the definition of the function. The number of transitions gives the number of times that the rules are used during the computation of the machine on a blank tape. In the last column, machines without references were analyzed by the author Mi09, MiWb.

Formally, a Turing machine $M=(Q, \Gamma, \delta)$ has a finite set of states $Q=\left\{q_{0}, q_{1}, \ldots, q_{n-1}\right\}$, a finite set of symbols $\Gamma=\{0,1, \ldots, m-1\}$, and a transition function (or next move function) $\delta$, which is a mapping

$$
\delta: Q \times \Gamma \rightarrow(\Gamma \times\{L, R\} \times Q) \cup\{(1, R, H)\} .
$$

If $\delta(q, a)=\left(b, D, q^{\prime}\right)$, then the Turing machine, when it is in state $q$ reading symbol $a$ on the current cell, writes symbol $b$ instead of $a$ on this cell, moves one cell left if $D=L$, one cell right if $D=R$, and enters state $q^{\prime}$. The transition function is usually given by a transition table. 


\begin{tabular}{|c|c|c|}
\hline Machine & Behavior & Study of behavior \\
\hline September 1997 & Pure Collatz-like $(4 \rightarrow 10)$ & \\
\hline Marxen and Buntrock & $\begin{array}{c}\text { without parameter } \\
5 \text { rules }\end{array}$ & Munafo MuWa \\
\hline$s>8.6 \times 10^{15}$ & 21 transitions & \\
\hline October 2000 & $\overline{\mathrm{R}}(\operatorname{bin}(\mathrm{p}))(2 \rightarrow 3)$ & \\
\hline Marxen and Buntrock & & \\
\hline (machine o) & 9 rules & \\
\hline$s>6.1 \times 10^{119}$ & 337 transitions & \\
\hline October 2000 & All $C(n)$ stop & \\
\hline Marxen and Buntrock & & Munafo MuWb \\
\hline (machine q) & 4 rules & Section 9 \\
\hline$s>6.1 \times 10^{925}$ & 5 transitions & \\
\hline March 2001 & $\mathrm{R}(\operatorname{bin}(\mathrm{p}))(2 \rightarrow 3)$ & \\
\hline Marxen and Buntrock & & Tooth To02] \\
\hline$s>3.0 \times 10^{1730}$ & $\begin{array}{c}20 \text { rules } \\
4911 \text { transitions }\end{array}$ & \\
\hline November 2007 & $\mathrm{R}(\operatorname{bin}(\mathrm{p}))(2 \rightarrow 5)$ & \\
\hline T. and S. Ligocki & & Section 8 \\
\hline$s>8.9 \times 10^{1762}$ & $\begin{array}{c}12 \text { rules } \\
3346 \text { transitions }\end{array}$ & \\
\hline December 2007 & $\overline{\mathrm{R}}(\operatorname{bin}(\mathrm{p}))(4 \rightarrow 6)$ & \\
\hline T. and S. Ligocki & 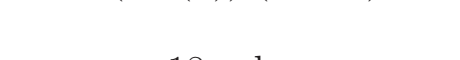 & \\
\hline$s>2.5 \times 10^{2879}$ & $\begin{array}{c}18 \text { rules } \\
11026 \text { transitions }\end{array}$ & \\
\hline May 2010 & Unclassifiable & \\
\hline Pavel Kropitz & & S. Ligocki Li10] \\
\hline$s>3.8 \times 10^{21132}$ & $\begin{array}{c}6 \text { rules } \\
22158 \text { transitions }\end{array}$ & Section 7 \\
\hline June 2010 & Exponential Collatz-like & \\
\hline $\begin{array}{l}\text { Pavel Kropitz } \\
s>7.4 \times 10^{36534}\end{array}$ & $\begin{array}{c}\text { without parameter } \\
4 \text { rules } \\
5 \text { transitions }\end{array}$ & Section 6 \\
\hline
\end{tabular}

Table 2: Study of behavior of $6 \times 2$ machines. See Def. 1.5 for the definition of exponential Collatz-like function. We write $\mathrm{R}(\operatorname{bin}(\mathrm{p}))$ when a potentially infinite set of rules would be needed for a complete analysis of the machine.

For greater convenience, the states of the Turing machines will be denoted by capital letters: $A, B, \ldots$ There is a special state $A$, called the initial state, and a special symbol 0 , called the blank symbol. In the busy beaver competition, at the beginning of a computation, the Turing machine is in state $A$, and the tape is blank, that is all the cells of the tape contain the blank symbol. There is another state $H$, the halting state, not in the set $Q$ of states. When a Turing machine enters this state, the computation stops. We impose that, at the last step, the machine writes 1 , moves right, and enters state $H$. The machines are normalized in the following way: When they are launched on a blank tape, they enter new states in the order $B, C, \ldots$, and they write new symbols in the order $1,2, \ldots$ 


\begin{tabular}{|c|c|c|}
\hline Machine & Behavior & Study of behavior \\
\hline $\begin{array}{l}\text { December } 2004 \\
\quad \text { Brady } \\
s=92,649,163\end{array}$ & $\begin{array}{c}\text { Pure Collatz-like }(2 \rightarrow 5) \\
\text { with parameter } \\
5 \text { rules } \\
11 \text { transitions }\end{array}$ & \\
\hline $\begin{array}{c}\text { July } 2005 \\
\text { Souris } \\
\sigma=36089 \\
s=310,341,163\end{array}$ & $\begin{array}{c}\text { Pure Collatz-like }(2 \rightarrow 5) \\
\text { with parameter } \\
5 \text { rules } \\
12 \text { transitions }\end{array}$ & \\
\hline $\begin{array}{l}\text { July } 2005 \\
\quad \text { Souris } \\
s=544,884,219\end{array}$ & $\begin{array}{c}\text { Pure Collatz-like }(3 \rightarrow 7) \\
\text { with parameter } \\
7 \text { rules } \\
12 \text { transitions }\end{array}$ & \\
\hline $\begin{array}{l}\text { August } 2005 \\
\text { Lafitte and Papazian } \\
\qquad s>4.9 \times 10^{9}\end{array}$ & $\begin{array}{c}\text { Pure collatz-like }(4 \rightarrow 7) \\
\text { with parameter } \\
8 \text { rules } \\
21 \text { transitions }\end{array}$ & \\
\hline $\begin{array}{l}\text { September } 2005 \\
\text { Lafitte and Papazian } \\
\qquad s>9.8 \times 10^{11}\end{array}$ & $\begin{array}{c}\text { Pure Collatz-like }(4 \rightarrow 7) \\
\text { with parameter } \\
7 \text { rules } \\
24 \text { transitions }\end{array}$ & \\
\hline $\begin{array}{c}\text { April } 2006 \\
\text { Lafitte and Papazian } \\
\qquad s>4.1 \times 10^{12}\end{array}$ & $\begin{array}{c}\text { Pure Collatz-like }(2 \rightarrow 5) \\
\text { with parameter } \\
5 \text { rules } \\
16 \text { transitions }\end{array}$ & \\
\hline $\begin{array}{l}\text { August } 2006 \\
\text { T. and S. Ligocki } \\
s>4.3 \times 10^{15}\end{array}$ & $\begin{array}{c}\text { Pure Collatz-like }(2 \rightarrow 5) \\
\text { with parameter } \\
4 \text { rules } \\
20 \text { transitions }\end{array}$ & S. Ligocki Li06b \\
\hline $\begin{array}{l}\text { November } 2007 \\
\text { T. and S. Ligocki } \\
\quad s>1.1 \times 10^{17}\end{array}$ & $\begin{array}{c}\text { Pure Collatz-like }(8 \rightarrow 14) \\
\text { without parameter } \\
9 \text { rules } \\
34 \text { transitions }\end{array}$ & Section 3 \\
\hline
\end{tabular}

Table 3: Study of behavior of $3 \times 3$ machines

A word is a finite string of symbols. The set of words with symbols in the set $\Gamma$ is denoted by $\Gamma^{*}$. The number of symbols in a word $x \in \Gamma^{*}$ is called the length of $x$ and is denoted by $|x|$. The empty word is the word of length zero, denoted by $\lambda$. If $x \in \Gamma^{*}$, and $n \geq 0, x^{n}$ is the word $x x \ldots x$, where $x$ is repeated $n$ times, that is, formally: $x^{0}=\lambda, x^{1}=x$ and $x^{n+1}=x^{n} x$. An infinite to the left string of 0 is denoted by ${ }^{\omega} 0$, and an infinite to the right string of 0 is denoted by $0^{\omega}$.

A configuration is a way to encode the symbols on the tape, the state, and the cell currently read by the head. The Turing machine is in configuration ${ }^{\omega} 0 x(S a) y 0^{\omega}$, with $S \in Q \cup\{H\}, a \in \Gamma, x, y \in \Gamma^{*}$, if the word $x a y$ is written on the tape, the state is $S$, and the head is reading symbol $a$. Since, at the beginning of the computation, the state is $A$ and the tape is blank, the initial configuration is ${ }^{\omega} 0(A 0) 0^{\omega}$. If the state is $H$, the configuration 


\begin{tabular}{|c|c|c|}
\hline Machine & Behavior & Study of behavior \\
\hline 1988 & Pure Collatz-like $(3 \rightarrow 5)$ & \\
Brady & with parameter & \\
$s=7195$ & 6 rules & \\
\hline February 2005 & 7 transitions & \\
T. and S. Ligocki & with parameter & Section 4 \\
$s=3,932,964$ & 7 rules & \\
\hline
\end{tabular}

Table 4: Study of behavior of $2 \times 4$ machines

\begin{tabular}{|c|c|c|}
\hline Machine & Behavior & Study of behavior \\
\hline October 2005 & Pure Collatz-like $(2 \rightarrow 5)$ & \\
\hline Lafitte, Papazian & with parameter & \\
\hline & 7 rules & \\
\hline$s>9.1 \times 10^{11}$ & 15 transitions & \\
\hline December 2005 & Pure Collatz-like $(2 \rightarrow 5)$ & \\
\hline Lafitte, Papazian & with parameter & \\
\hline & 5 rules & \\
\hline$s>9.2 \times 10^{11}$ & 14 transitions & \\
\hline May 2006 & Pure Collatz-like $(3 \rightarrow 4)$ & \\
\hline Lafitte, Papazian & with parameter & \\
\hline & 7 rules & \\
\hline$s>3.7 \times 10^{12}$ & 45 transitions & \\
\hline June 2006 & Pure Collatz-like $(2 \rightarrow 3)$ & \\
\hline Lafitte, Papazian & with parameter & \\
\hline$s>1.4 \times 10^{13}$ & 36 transitions & \\
\hline August 2006 & Pure Collatz-like $(2 \rightarrow 5)$ & \\
\hline T. and S. Ligocki & with parameter & S. Ligocki [Li06a] \\
\hline$s>7.0 \times 10^{21}$ & 30 transitions & \\
\hline November 2007 & Pure Collatz-like $(2 \rightarrow 3)$ & \\
\hline T. and S. Ligocki & with parameter & Section 5 \\
\hline$s>1.9 \times 10^{704}$ & 2002 transitions & \\
\hline
\end{tabular}

Table 5: Study of behavior of $2 \times 5$ machines

is halting. We also consider configurations $x(S a) y$ with finite length. If the computation from configuration $C_{1}$ to configuration $C_{2}$ takes $t$ steps, we write $C_{1} \vdash(t) C_{2}$, and $t$ is said to be the time taken by the machine to go from $C_{1}$ to $C_{2}$. If $C_{2}$ is a halting configuration, we also write $C_{1} \vdash(t)$ END. We write $C_{1} \vdash() C_{2}$ if the time is not specified. If $C_{1}$ and $C_{2}$ are configurations with finite length, then they refer to the same part of the tape. For example, $(A 0) 0 \vdash(1) 1(B 0)$ if $\delta(A, 0)=(1, R, B)$. 


\begin{tabular}{|c|c|c|c|}
\hline$M_{1}$ & 0 & 1 & 2 \\
\hline$A$ & $1 \mathrm{RB}$ & $2 \mathrm{LA}$ & $1 \mathrm{LC}$ \\
\hline$B$ & $0 \mathrm{LA}$ & $2 \mathrm{RB}$ & $1 \mathrm{LB}$ \\
\hline$C$ & $1 \mathrm{RH}$ & $1 \mathrm{RA}$ & $1 \mathrm{RC}$ \\
\hline
\end{tabular}

Table 6: Machine $M_{1}$ discovered in November 2007 by T. and S. Ligocki. Such tables are read as in the following example. When machine $M_{1}$ is in state $A$ and reads symbol 0 , then it writes symbol 1 instead of symbol 0 , moves one cell to the right, and enters state $B$.

A Turing machine $M$ computes a partial function $f_{M}: \Gamma^{*} \rightarrow \Gamma^{*}$ as follows. let $x=$ $x_{1} \ldots x_{n} \in \Gamma^{*}$. Then $x$ becomes an input for $M$ by considering the computation of $M$ on initial configuration ${ }^{\omega} 0\left(A x_{1}\right) x_{2} \ldots x_{n} 0^{\omega}$. If $M$ never stops on this configuration, then $f_{M}(x)$ is undefined. If $M$ stops, in configuration ${ }^{\omega} 0 y(H a) z 0^{\omega}$, with $a \in \Gamma, y, z \in \Gamma^{*}$, then the output $f_{M}(x)$ is defined from this configuration by a suitable convention. The halting set is $\left\{x \in \Gamma^{*}: f_{M}(x)\right.$ is defined $\}$. The halting problem for machine $M$ is the problem consisting in determining the halting set. Note that the Turing machines with two symbols 0 and 1 are powerful enough to compute any computable function, and their halting sets can be any computably enumerable (also called recursively enumerable) set.

A Turing machine with $n$ states and $m$ symbols is called a $n \times m$ machine. The set of $n \times m$ machines is denoted by $\operatorname{TM}(n, m)$. With our definition of the transition function, there are $(2 n m+1)^{n m}$ machines in the set $\operatorname{TM}(n, m)$. In the busy beaver competition, for fixed numbers of states $n$ and symbols $m$, all the $(2 n m+1)^{n m}$ Turing machines in $\operatorname{TM}(n, m)$ are launched on the blank tape. Some of them never stop. Those which stop are called busy beaver. Each busy beaver takes some time to stop, and leaves some non-blank symbols on the tape, so busy beavers are involved in two competitions: to take the longest time before stopping, and to leave the greatest number of non-blank symbols on the tape when stopping. The time taken by Turing machine $M$ to stop is denoted by $s(M)$, and the number of nonblank symbols left by $M$ when it stops is denoted by $\sigma(M)$. The busy beaver functions are defined by

$$
\begin{aligned}
& S(n, m)=\max \{s(M): M \text { is a busy beaver with } n \text { states and } m \text { symbols }\} \\
& \Sigma(n, m)=\max \{\sigma(M): M \text { is a busy beaver with } n \text { states and } m \text { symbols }\}
\end{aligned}
$$

Rado Ra62 initially defined functions $S(n)=S(n, 2)$ and $\Sigma(n)=\Sigma(n, 2)$ for Turing machines with $n$ states and two symbols.

\section{Pure Collatz-like Behavior}

Let $M_{1}$ be the $3 \times 3$ Turing machine defined by Table 6

We have $s\left(M_{1}\right)=119,112,334,170,342,540$ and $\sigma\left(M_{1}\right)=374,676,383$.

This machine is the current champion for the busy beaver competition for $3 \times 3$ machines. It was discovered in November 2007 by Terry and Shawn Ligocki, who wrote (email on November, 9th) that they enumerated all the $3 \times 3$ machines and applied the techniques of acceleration and proof systems originally developed by Marxen and Buntrock.

The following theorem gives the rules that enable Turing machine $M_{1}$ to reach a halting configuration from a blank tape. 
Theorem 3.1. We have the following transitions between configurations of Turing machine $M_{1}$. Let $C(n)={ }^{\omega} 0(A 0) 2^{n} 0^{\omega}$. Then

(a) ${ }^{\omega} 0(A 0) 0^{\omega} \quad \vdash(3) \quad C(1)$,

and, for all $k \geq 0$,

(b) $C(8 k+1) \vdash\left(112 k^{2}+116 k+13\right) \quad C(14 k+3)$,

(c) $C(8 k+2) \vdash\left(112 k^{2}+144 k+38\right) \quad C(14 k+7)$,

(d) $C(8 k+3) \vdash\left(112 k^{2}+172 k+54\right) \quad C(14 k+8)$,

(e) $C(8 k+4) \vdash\left(112 k^{2}+200 k+74\right) \quad C(14 k+9)$,

(f) $C(8 k+5) \vdash\left(112 k^{2}+228 k+97\right) \quad{ }^{\omega} 01(H 1) 2^{14 k+9} 0^{\omega}$,

(g) $C(8 k+6) \vdash\left(112 k^{2}+256 k+139\right) \quad C(14 k+14)$,

(h) $C(8 k+7) \vdash\left(112 k^{2}+284 k+169\right) \quad C(14 k+15)$,

(i) $C(8 k+8) \vdash\left(112 k^{2}+312 k+203\right) \quad C(14 k+16)$.

Proof. A direct inspection of the transition table gives

$\begin{array}{llll}(1) & 0(A 0) 0 & \vdash(3) & (A 0) 20, \\ (2) & 0^{3}(A 0) 2^{5} & \vdash(53) & (B 1) 1^{8}, \\ (3) & 0(A 1) & \vdash(1) & (A 0) 2, \\ (4) & 1(A 1) & \vdash(1) & (A 1) 2, \\ (5) & 02(A 1) & \vdash(3) & 1(H 1) 2, \\ (6) & 12(A 1) & \vdash(4) & (A 1) 22, \\ (7) & 22(A 1) & \vdash(8) & (A 1) 22, \\ (8) & 2(B 1) 0^{2} & \vdash(7) & 11(A 1), \\ (9) & (B 1) 1 & \vdash(1) & 2(B 1), \\ (10) & (B 1) 2 & \vdash(1) & 2(B 2), \\ (11) & 0^{3}(B 2) & \vdash(14) & 1^{3}(B 1), \\ (12) & 1(B 2) & \vdash(1) & (B 1) 1, \\ (13) & 2(B 2) & \vdash(1) & (B 2) 1 .\end{array}$

From this point, $k$ will be an integer, $k \geq 0$.

Iterating, respectively, (4), (7), (9) and (13) gives

$$
\begin{array}{llll}
(14) & 1^{k}(A 1) & \vdash(k) & (A 1) 2^{k}, \\
(15) & 2^{2 k}(A 1) & \vdash(8 k) & (A 1) 2^{2 k}, \\
(16) & (B 1) 1^{k} & \vdash(k) & 2^{k}(B 1), \\
(17) & 2^{k}(B 2) & \vdash(k) & (B 2) 1^{k} .
\end{array}
$$

Using consecutively (16), (10), (17) and (12), we get

(18) $1(B 1) 1^{k} 2 \vdash(2 k+3) \quad(B 1) 1^{k+2}$.

Using (16), (10), (17) and (11), we get

(19) $0^{3}(B 1) 1^{k} 2 \quad \vdash(2 k+16) \quad 1^{3}(B 1) 1^{k+1}$.

Using (19) and three times (18), we get

(20) $0^{3}(B 1) 1^{k} 2^{4} \vdash(8 k+43) \quad(B 1) 1^{k+7}$.

For any $n \geq 0$, by induction on $k$, using (20), we get

$(21) \quad 0^{3 k}(B 1) 1^{n} 2^{4 k} \quad \vdash\left(28 k^{2}+(8 n+15) k\right) \quad(B 1) 1^{7 k+n}$.

By taking $n=8$ in $(21)$, we get

$(22) \quad 0^{3 k}(B 1) 1^{8} 2^{4 k} \vdash\left(28 k^{2}+79 k\right) \quad(B 1) 1^{7 k+8}$.

Using (8), (14) and (15), we get

(23) $2^{2 k+1}(B 1) 0^{2} \quad \vdash(8 k+9) \quad(A 1) 2^{2 k+2} 0$.

We are now ready to prove the results of the theorem. 
Using (2), (22) and (16), we get

(24) $0^{3 k+3}(A 0) 2^{4 k+5} \quad \vdash\left(28 k^{2}+86 k+61\right) \quad 2^{7 k+8}(B 1)$.

Using (24), (23) and (5) we get

$(25) \quad 0^{6 k+4}(A 0) 2^{8 k+5} 0^{2} \vdash\left(112 k^{2}+228 k+97\right) \quad 1(H 1) 2^{14 k+9} 0$.

Using (24), (23) and (3) we get

$$
0^{6 k+7}(A 0) 2^{8 k+9} 0^{2} \quad \vdash\left(112 k^{2}+340 k+241\right) \quad(A 0) 2^{14 k+17} 0,
$$

and this result is still true for $k=-1$, so we have

(26) $0^{6 k+1}(A 0) 2^{8 k+1} 0^{2} \vdash\left(112 k^{2}+116 k+13\right) \quad(A 0) 2^{14 k+3} 0$.

Using (2), (22), (19) and (16) we get

(27) $0^{3 k+6}(A 0) 2^{4 k+6} \vdash\left(28 k^{2}+100 k+94\right) \quad 1^{3} 2^{7 k+9}(B 1)$.

Using (27), (23), (14) and (3) we get

$(28) \quad 0^{6 k+7}(A 0) 2^{8 k+6} 0^{2} \quad \vdash\left(112 k^{2}+256 k+139\right) \quad(A 0) 2^{14 k+14} 0$.

Using (27), (23), (6), (14) and (3) we get

$$
0^{6 k+10}(A 0) 2^{8 k+10} 0^{2} \quad \vdash\left(112 k^{2}+368 k+294\right) \quad(A 0) 2^{14 k+21} 0,
$$

and this result is still true for $k=-1$, so we have

(29) $0^{6 k+4}(A 0) 2^{8 k+2} 0^{2} \quad \vdash\left(112 k^{2}+144 k+38\right) \quad(A 0) 2^{14 k+7} 0$.

Using (2), (22), (19), (18), (16), (8) and (14) we get

(30) $\quad 0^{3 k+6}(A 0) 2^{4 k+7} 0^{2} \quad \vdash\left(28 k^{2}+114 k+126\right) \quad 1^{2} 2^{7 k+10}(A 1) 220$.

Using (30), (15), (14) and (3) we get

(31) $0^{6 k+7}(A 0) 2^{8 k+7} 0^{2} \vdash\left(112 k^{2}+284 k+169\right) \quad(A 0) 2^{14 k+15} 0$.

Using (30), (15), (6), (4) and (3) we get

$0^{6 k+10}(A 0) 2^{8 k+11} 0^{2} \quad \vdash\left(112 k^{2}+396 k+338\right) \quad(A 0) 2^{14 k+22} 0$,

and this result is still true for $k=-1$, so we have

(32) $0^{6 k+4}(A 0) 2^{8 k+3} 0^{2} \quad \vdash\left(112 k^{2}+172 k+54\right) \quad(A 0) 2^{14 k+8} 0$.

Using (2), (22), (19), (18), (18), (16), (8) and (14) we get

(33) $0^{3 k+6}(A 0) 2^{4 k+8} 0^{2} \quad \vdash\left(28 k^{2}+128 k+153\right) \quad 12^{7 k+12}(A 1) 220$.

Using (33), (15), (4) and (3) we get

(34) $0^{6 k+7}(A 0) 2^{8 k+8} 0^{2} \quad \vdash\left(112 k^{2}+312 k+203\right) \quad(A 0) 2^{14 k+16} 0$.

Using (33), (15), (6) and (3) we get

$$
0^{6 k+10}(A 0) 2^{8 k+12} 0^{2} \quad \vdash\left(112 k^{2}+424 k+386\right) \quad(A 0) 2^{14 k+23} 0,
$$

and this result is still true for $k=-1$, so we have

(35) $\quad 0^{6 k+4}(A 0) 2^{8 k+4} 0^{2} \quad \vdash\left(112 k^{2}+200 k+74\right) \quad(A 0) 2^{14 k+9} 0$.

The results (1), (26), (29), (32), (35), (25), (28), (31) and (34) give, respectively, the results (a) $-(\mathrm{i})$ of the theorem.

Using the rules of this theorem, we have, in 34 transitions,

$$
{ }^{\omega} 0(A 0) 0^{\omega} \vdash(3) \quad C(1) \vdash(13) \quad C(3) \vdash() \cdots \vdash() \quad{ }^{\omega} 01(H 1) 2^{374,676,381} 0^{\omega} .
$$

Let us try to give an informal explanation of how Turing machine $M_{1}$ works. Note that this explanation will also apply to the machines $M_{2}$ and $M_{3}$ below. The two main ideas are the following ones. First, a string of symbols 2 is nibbled four by four, and each time a string of four symbols 2 is nibbled, a string of seven symbols 2 is potentially created (see transitions (20) and (21) in the proof of Theorem 3.1). This explains the type $8 \rightarrow 14$ of the simulated Collatz-like function. Second, there are edge effects when the ends of a string of symbols 2 are reached, which explain the multiplicity of cases. 


\begin{tabular}{|c|c|c|c|c|}
\hline$M_{2}$ & 0 & 1 & 2 & 3 \\
\hline$A$ & $1 \mathrm{RB}$ & $2 \mathrm{LA}$ & $1 \mathrm{RA}$ & $1 \mathrm{RA}$ \\
\hline$B$ & $1 \mathrm{LB}$ & $1 \mathrm{LA}$ & $3 \mathrm{RB}$ & $1 \mathrm{RH}$ \\
\hline
\end{tabular}

Table 7: Machine $M_{2}$ discovered in February 2005 by T. and S. Ligocki

Let $g_{1}$ be the pure Collatz-like function defined by: for $k \geq 0$,

$$
\begin{aligned}
& g_{1}(8 k+1)=14 k+3, \\
& g_{1}(8 k+2)=14 k+7, \\
& g_{1}(8 k+3)=14 k+8, \\
& g_{1}(8 k+4)=14 k+9, \\
& g_{1}(8 k+5)=\text { undefined, } \\
& g_{1}(8 k+6)=14 k+14, \\
& g_{1}(8 k+7)=14 k+15, \\
& g_{1}(8 k+8)=14 k+16 .
\end{aligned}
$$

Then $g_{1}^{33}(1)$ is undefined.

The theorem gives immediately the following proposition.

Proposition 3.2. The behavior of Turing machine $M_{1}$, on inputs $02^{n}, n \geq 1$, depends on the behavior of iterated $g_{1}^{k}(n), k \geq 1$.

Since the behavior of iterated $g_{1}^{k}(n)$ is an open problem in mathematics, this is also the case for the halting problem for Turing machine $M_{1}$.

Let $h_{1}(n)=\min \left\{k: g_{1}^{k}(n)\right.$ is undefined $\}$. We have seen that $h_{1}(1)=33$. We also have $h_{1}(144)=41, h_{1}(270)=51$.

\section{Collatz-Like With Parameter: first eXample}

Let $M_{2}$ be the $2 \times 4$ Turing machine defined by Table 7 .

We have $s\left(M_{2}\right)=3,932,964$ and $\sigma\left(M_{2}\right)=2050$.

This machine is the current champion for the busy beaver competition for $2 \times 4$ machines. It was discovered in February 2005 by Terry and Shawn Ligocki, who wrote (email on February, 13th) that they found this machine using simulated annealing.

The following theorem gives the rules that enable Turing machine $M_{2}$ to reach a halting configuration from a blank tape.

Theorem 4.1. We have the following transitions between configurations of Turing machine $M_{2}$. Let

$$
\begin{aligned}
& C(n, 1)={ }^{\omega} 0(A 0) 2^{n} 10^{\omega}, \\
& C(n, 2)={ }^{\omega} 0(A 0) 2^{n} 110^{\omega} . \\
& \text { Then } \\
& (a) \omega^{\omega} 0(A 0) 0^{\omega} \vdash(6) \quad C(1,2), \\
& \text { and, for all } k \geq 0,
\end{aligned}
$$



(b) $C(3 k, 1) \quad \vdash\left(15 k^{2}+9 k+3\right) \quad C(5 k+1,1)$,
(c) $C(3 k+1,1) \vdash\left(15 k^{2}+24 k+13\right){ }^{\omega} 013^{5 k+2} 1(H 1) 0^{\omega}$,
(d) $C(3 k+2,1) \vdash\left(15 k^{2}+29 k+17\right) \quad C(5 k+4,2)$,
(e) $C(3 k, 2) \quad \vdash\left(15 k^{2}+11 k+3\right) \quad C(5 k+1,2)$,
(f) $C(3 k+1,2) \vdash\left(15 k^{2}+21 k+7\right) \quad C(5 k+3,1)$,
(g) $C(3 k+2,2) \vdash\left(15 k^{2}+36 k+23\right){ }^{\omega} 013^{5 k+4} 1(H 1) 0^{\omega}$.

Proof. A direct inspection of the transition table gives
(1) $0^{2}(A 0) 0 \vdash(6) \quad(A 0) 211$,
(2) $1(A 0) 0 \quad \vdash(3) \quad(A 1) 11$,
(3) $(A 0) 2 \quad \vdash(1) \quad 1(B 2)$,
(4) $0(A 1) \quad \vdash(1) \quad(A 0) 2$,
(5) $1(A 1) \quad \vdash(1) \quad(A 1) 2$,
(6) $3(A 1) \quad \vdash(2) \quad 1(A 2)$,
(7) $(A 2) 0 \quad \vdash(1) 1(A 0)$,
(8) $\quad(A 2) 1 \quad \vdash(1) \quad 1(A 1)$,
(9) $(A 2) 2 \quad \vdash(1) \quad 1(A 2)$,
(10) $(B 2) 0 \quad \vdash(3) \quad 1(H 1)$,
(11) $(B 2) 1 \quad \vdash(4) \quad(A 1) 2$,
(12) $(B 2) 2 \quad \vdash(1) \quad 3(B 2)$.

Iterating, respectively, (5), (9) and (12) gives

$$
\begin{array}{llll}
(13) & 1^{k}(A 1) & \vdash(k) & (A 1) 2^{k}, \\
(14) & (A 2) 2^{k} & \vdash(k) & 1^{k}(A 2), \\
(15) & (B 2) 2^{k} & \vdash(k) & 3^{k}(B 2) .
\end{array}
$$

Using (2), (13) and (4) we get

$$
01^{k+1}(A 0) 0 \vdash(k+4) \quad(A 0) 2^{k+1} 11,
$$

and this result is still true for $k=-1$, so we have

(16) $\quad 01^{k}(A 0) 0 \quad \vdash(k+3) \quad(A 0) 2^{k} 11$.

Using (2), (13), (6), (14), (8), (13) and (4) we get

(17) $0131^{k+1}(A 0) 0 \quad \vdash(3 k+10) \quad(A 0) 2^{k+4} 1$.

Using (2), (13), (6), (14), (8), (13), (6), (14), (8), (13) and (4) we get

(18) $01331^{k+1}(A 0) 0 \vdash(5 k+19) \quad(A 0) 2^{k+6}$.

Using (3), (15) and (10) we get

(19) $(A 0) 2^{k+1} 0 \quad \vdash(k+4) \quad 13^{k} 1(H 1)$.

Using (3), (15), (11), (6), (9) and (7) we get

(20) $(A 0) 2^{k+2} 10 \vdash(k+10) \quad 13^{k} 1^{3}(A 0)$.

Using (3), (15), (11), (6), (9), (8), (13), (6), (14) and (7) we get

(21) $(A 0) 2^{k+3} 110 \quad \vdash(k+20) \quad 13^{k} 1^{5}(A 0)$.

Using (2), (13), (6), (14), (8), (13), (6), (14), (8), (13), (6), (14) and (7) we get

(22) $3^{3} 1^{k+1}(A 0) 0^{2} \quad \vdash(6 k+24) \quad 1^{k+6}(A 0)$.

By induction on $k$, from $(22)$, we get

(23) $\quad 3^{3 k} 1^{n+1}(A 0) 0^{2 k} \quad \vdash\left(15 k^{2}+6 n k+9 k\right) \quad 1^{5 k+n+1}(A 0)$,

so we have, for $n=2$ and $n=4$

$$
\begin{array}{llll}
(24) & 3^{3 k} 1^{3}(A 0) 0^{2 k} & \vdash\left(15 k^{2}+21 k\right) & 1^{5 k+3}(A 0), \\
(25) & 3^{3 k} 1^{5}(A 0) 0^{2 k} & \vdash\left(15 k^{2}+33 k\right) & 1^{5 k+5}(A 0) .
\end{array}
$$

We are now ready to prove the theorem. 
Using (20), (24) and (17) we get

$$
0(A 0) 2^{3 k+3} 10^{2 k+2} \vdash\left(15 k^{2}+39 k+27\right) \quad(A 0) 2^{5 k+6} 1,
$$

and the result is still true for $k=-1$, so we have

$$
\text { (26) } 0(A 0) 2^{3 k} 10^{2 k} \vdash\left(15 k^{2}+9 k+3\right) \quad(A 0) 2^{5 k+1} 1 .
$$

Using (20), (24), (18) and (19) we get

$$
0(A 0) 2^{3 k+4} 10^{2 k+3} \vdash\left(15 k^{2}+54 k+52\right) \quad 13^{5 k+7} 1(H 1),
$$

and the result is still true for $k=-1$, so we have

$$
\text { (27) } 0(A 0) 2^{3 k+1} 10^{2 k+1} \vdash\left(15 k^{2}+24 k+13\right) \quad 13^{5 k+2} 1(H 1) \text {. }
$$

Using (20), (24) and (16) we get

$$
\text { (28) } 0(A 0) 2^{3 k+2} 10^{2 k+2} \vdash\left(15 k^{2}+29 k+17\right) \quad(A 0) 2^{5 k+4} 11 \text {. }
$$

Using (21), (25) and (16) we get

$$
0(A 0) 2^{3 k+3} 110^{2 k+2} \vdash\left(15 k^{2}+41 k+29\right) \quad(A 0) 2^{5 k+6} 11,
$$

and the result is still true for $k=-1$, so we have

$$
\text { (29) } 0(A 0) 2^{3 k} 110^{2 k} \vdash\left(15 k^{2}+11 k+3\right) \quad(A 0) 2^{5 k+1} 11 .
$$

Using (21), (25) and (17) we get

$$
0(A 0) 2^{3 k+4} 110^{2 k+2} \vdash\left(15 k^{2}+51 k+43\right) \quad(A 0) 2^{5 k+8} 1,
$$

and the result is still true for $k=-1$, so we have

$$
\text { (30) } 0(A 0) 2^{3 k+1} 110^{2 k} \vdash\left(15 k^{2}+21 k+7\right) \quad(A 0) 2^{5 k+3} 1 \text {. }
$$

Using (21), (25), (18) and (19) we get

$$
0(A 0) 2^{3 k+5} 110^{2 k+3} \vdash\left(15 k^{2}+66 k+74\right) \quad 13^{5 k+9} 1(H 1),
$$

and the result is still true for $k=-1$, so we have

$$
\text { (31) } 0(A 0) 2^{3 k+2} 110^{2 k+1} \vdash\left(15 k^{2}+36 k+23\right) \quad 13^{5 k+4} 1(H 1) .
$$

The theorem comes from results (1) and (26)-(31).

Using the rules of this theorem, we have, in 14 transitions,

$$
{ }^{\omega} 0(A 0) 0^{\omega} \vdash(6) \quad C(1,2) \vdash(7) \quad C(3,1) \vdash() \cdots \vdash() \quad{ }^{\omega} 013^{2047} 1(H 1) 0^{\omega} .
$$

Informally, Turing machine $M_{2}$ works according to the same ideas as Turing machine $M_{1}$ does, but, in addition, edge effects depend also on the parameter.

Let $g_{2}$ be the pure Collatz-like function with parameter defined by: for $k \geq 0$,

$$
\begin{aligned}
g_{2}(3 k, 1)= & (5 k+1,1), \\
g_{2}(3 k+1,1)= & \text { undefined, } \\
g_{2}(3 k+2,1)= & (5 k+4,2), \\
g_{2}(3 k, 2)= & (5 k+1,2), \\
g_{2}(3 k+1,2)= & (5 k+3,1), \\
g_{2}(3 k+2,2)= & \text { undefined. }
\end{aligned}
$$

Then $g_{2}^{13}(1,2)$ is undefined.

The theorem gives immediately the following proposition.

Proposition 4.2. The behavior of Turing machine $M_{2}$, on inputs $02^{n} 1^{i}, n \geq 1, i \in\{1,2\}$ depends on the behavior of iterated $g_{2}^{k}(n, i), k \geq 1$.

Since the behavior of iterated $g_{2}^{k}(n, i)$ is an open problem in mathematics, this is also the case for the halting problem for Turing machine $M_{2}$.

Let $h_{2}(n, i)=\min \left\{k: g_{2}^{k}(n, i)\right.$ is undefined $\}$. We have seen that $h_{2}(1,2)=13$. We also have $h_{2}(137,1)=16, h_{2}(210,2)=20$. 


\begin{tabular}{|c|c|c|c|c|c|}
\hline$M_{3}$ & 0 & 1 & 2 & 3 & 4 \\
\hline$A$ & $1 \mathrm{RB}$ & $2 \mathrm{LA}$ & $1 \mathrm{RA}$ & $2 \mathrm{LB}$ & $2 \mathrm{LA}$ \\
\hline$B$ & $0 \mathrm{LA}$ & $2 \mathrm{RB}$ & $3 \mathrm{RB}$ & $4 \mathrm{RA}$ & $1 \mathrm{RH}$ \\
\hline
\end{tabular}

Table 8: Machine $M_{3}$ discovered in November 2007 by T. and S. Ligocki

\section{Collatz-Like With Parameter: Second example}

Let $M_{3}$ be the $2 \times 5$ Turing machine defined by Table 8 ,

We have $s\left(M_{3}\right)>1.9 \times 10^{704}$ and $\sigma\left(M_{3}\right)>1.7 \times 10^{352}$.

This machine is the current champion for the busy beaver competition for $2 \times 5$ machines. It was discovered in November 2007 by Terry and Shawn Ligocki, who wrote (email on November, 9th) that, as they did for $3 \times 3$ machine $M_{1}$, they enumerated all the $2 \times 5$ machines and applied the techniques of acceleration and proof systems originally developed by Marxen and Buntrock.

The following theorem gives the rules that enable Turing machine $M_{3}$ to reach a halting configuration from a blank tape.

Theorem 5.1. We have the following transitions between configurations of Turing machine $M_{3}$. Let

$$
\begin{aligned}
& C(n, 1)={ }^{\omega} 013^{n}(B 0) 0^{\omega} \\
& C(n, 2)={ }^{\omega} 023^{n}(B 0) 0^{\omega} \\
& C(n, 3)={ }^{\omega} 03^{n}(B 0) 0^{\omega} \\
& C(n, 4)={ }^{\omega} 04113^{n}(B 0) 0^{\omega} \\
& C(n, 5)={ }^{\omega} 04123^{n}(B 0) 0^{\omega} \\
& C(n, 6)={ }^{\omega} 0413^{n}(B 0) 0^{\omega} \\
& C(n, 7)={ }^{\omega} 0423^{n}(B 0) 0^{\omega} \\
& C(n, 8)={ }^{\omega} 043^{n}(B 0) 0^{\omega} .
\end{aligned}
$$

Then

$$
\begin{aligned}
& \text { (a) }{ }^{\omega} 0(A 0) 0^{\omega} \quad \vdash(1) \quad C(0,1), \\
& \text { and, for all } k \geq 0 \text {, } \\
& \text { (b) } C(2 k, 1) \quad \vdash\left(3 k^{2}+8 k+4\right) \quad C(3 k+1,1) \text {, } \\
& \text { (c) } C(2 k, 2) \quad \vdash\left(3 k^{2}+14 k+9\right) \quad C(3 k+2,1) \text {, } \\
& \text { (d) } C(2 k, 3) \quad \vdash\left(3 k^{2}+8 k+2\right) \quad C(3 k, 1) \text {, } \\
& \text { (e) } C(2 k, 4) \quad \vdash\left(3 k^{2}+8 k+8\right) \quad C(3 k+3,1) \text {, } \\
& \text { (f) } C(2 k, 5) \quad \vdash\left(3 k^{2}+14 k+13\right) \quad C(3 k+4,1) \text {, } \\
& \text { (g) } C(2 k, 6) \quad \vdash\left(3 k^{2}+8 k+6\right) \quad C(3 k+2,1) \text {, } \\
& \text { (h) } C(2 k, 7) \quad \vdash\left(3 k^{2}+14 k+11\right) \quad C(3 k+3,1) \text {, } \\
& \text { (i) } C(2 k, 8) \quad \vdash\left(3 k^{2}+8 k+4\right) \quad C(3 k+1,1) \text {, } \\
& \text { (j) } C(2 k+1,1) \vdash\left(3 k^{2}+8 k+4\right) \quad C(3 k+1,2) \text {, } \\
& \text { (k) } C(2 k+1,2) \quad \vdash\left(3 k^{2}+8 k+4\right) \quad C(3 k+2,3) \text {, } \\
& \text { (l) } C(2 k+1,3) \quad \vdash\left(3 k^{2}+8 k+22\right) \quad C(3 k+1,4) \text {, } \\
& \text { (m) } C(2 k+1,4) \vdash\left(3 k^{2}+8 k+4\right) \quad C(3 k+1,5) \text {, } \\
& \text { (n) } C(2 k+1,5) \vdash\left(3 k^{2}+8 k+4\right) \quad C(3 k+2,6) \text {, } \\
& \text { (o) } C(2 k+1,6) \vdash\left(3 k^{2}+8 k+4\right) \quad C(3 k+1,7) \text {, } \\
& \text { (p) } C(2 k+1,7) \vdash\left(3 k^{2}+8 k+4\right) \quad C(3 k+2,8) \text {, } \\
& \text { (q) } C(2 k+1,8) \vdash\left(3 k^{2}+5 k+3\right) \quad{ }^{\omega} 01(H 2) 2^{3 k} 0^{\omega} .
\end{aligned}
$$

Proof. A direct inspection of the transition table gives 


$\begin{array}{llll}(1) & (A 0) 0 & \vdash(1) & 1(B 0), \\ (2) & 0(A 0) 0 & \vdash(17) & 41(A 0), \\ (3) & (A 0) 2 & \vdash(1) & 1(B 2), \\ (4) & 0(A 1) & \vdash(1) & (A 0) 2, \\ (5) & 1(A 1) & \vdash(1) & (A 1) 2, \\ (6) & 4(A 1) & \vdash(1) & (A 4) 2, \\ (7) & (A 2) 0^{2} & \vdash(2) & 1^{2}(B 0), \\ (8) & (A 2) 2 & \vdash(1) & 1(A 2), \\ (9) & 1(B 0) & \vdash(1) & (A 1) 0, \\ (10) & 3^{2}(B 0) 0 & \vdash(5) & 41^{2}(B 0), \\ (11) & (B 2) 0 & \vdash(1) & 3(B 0), \\ (12) & (B 2) 2 & \vdash(1) & 3(B 2), \\ (13) & 0(A 4) & \vdash(1) & (A 0) 2, \\ (14) & 1(A 4) & \vdash(1) & (A 1) 2, \\ (15) & 2(A 4) & \vdash(1) & (A 2) 2, \\ (16) & 0^{2} 3(A 4) & \vdash(3) & (A 0) 02^{2}, \\ (17) & 13(A 4) & \vdash(4) & 23(B 2), \\ (18) & 23(A 4) & \vdash(4) & 33(B 2), \\ (19) & 3^{2}(A 4) & \vdash(4) & 41(A 2), \\ (20) & 43(A 4) & \vdash(3) & 1(H 2) 2, \\ (21) & 04(A 4) & \vdash(2) & (A 0) 2^{2} .\end{array}$

Iterating, respectively, (5), (8) and (12) gives

(22) $1^{k}(A 1) \quad \vdash(k) \quad(A 1) 2^{k}$,

(23) $\quad(A 2) 2^{k} \vdash(k) \quad 1^{k}(A 2)$,

(24) $\quad(B 2) 2^{k} \vdash(k) \quad 3^{k}(B 2)$.

Using (9), (22), (6), (19), (23) and (7) we get

$3^{2} 41^{k+1}(B 0) 0 \vdash(2 k+9) \quad 41^{k+4}(B 0)$,

and the result is still true for $k=-1$, so we have

(25) $\quad 3^{2} 41^{k}(B 0) 0 \quad \vdash(2 k+7) \quad 41^{k+3}(B 0)$.

For any $n \geq 0$, by induction on $k$, using (25), we get

(26) $\quad 3^{2 k} 41^{n}(B 0) 0^{k} \vdash\left(3 k^{2}+(2 n+4) k\right) \quad 41^{3 k+n}(B 0)$,

so we have, for $n=2$ in $(26)$,

(27) $3^{2 k} 41^{2}(B 0) 0^{k} \vdash\left(3 k^{2}+8 k\right) \quad 41^{3 k+2}(B 0)$.

Using (10), (27), (9), (22) and (6), we get

(28) $3^{2 k+2}(B 0) 0^{k+1} \vdash\left(3 k^{2}+11 k+8\right) \quad(A 4) 2^{3 k+2} 0$.

Using (3), (24) and (11) we get

$(A 0) 2^{k+1} 0 \quad \vdash(k+2) \quad 13^{k+1}(B 0)$,

and the result is still true for $k=-1$, so we have

(29) $(A 0) 2^{k} 0 \vdash(k+1) \quad 13^{k}(B 0)$.

Using (15), (23), (7), (9) and (22), we get

(30) $2(A 4) 2^{k} 0^{2} \vdash(2 k+7) \quad(A 1) 2^{k+2} 0$.

We are now ready to prove the theorem.

Using (28), (14), (4) and (29) we get

$013^{2 k+2}(B 0) 0^{k+1} \quad \vdash\left(3 k^{2}+14 k+15\right) \quad 13^{3 k+4}(B 0)$,

and the result is still true for $k=-1$, so we have

(31) $013^{2 k}(B 0) 0^{k} \vdash\left(3 k^{2}+8 k+4\right) \quad 13^{3 k+1}(B 0)$. 
Using (28), (30), (4) and (29) we get

$023^{2 k+2}(B 0) 0^{k+2} \vdash\left(3 k^{2}+20 k+26\right) \quad 13^{3 k+5}(B 0)$,

and the result is still true for $k=-1$, so we have

(32) $023^{2 k}(B 0) 0^{k+1} \vdash\left(3 k^{2}+14 k+9\right) \quad 13^{3 k+2}(B 0)$.

Using (28), (13) and (29) we get

$03^{2 k+2}(B 0) 0^{k+1} \vdash\left(3 k^{2}+14 k+13\right) \quad 13^{3 k+3}(B 0)$,

and the result is still true for $k=-1$, so we have

(33) $03^{2 k}(B 0) 0^{k} \quad \vdash\left(3 k^{2}+8 k+2\right) \quad 13^{3 k}(B 0)$.

Using (28), (14), (5), (6), (13) and (29) we get

$04113^{2 k+2}(B 0) 0^{k+1} \vdash\left(3 k^{2}+14 k+19\right) \quad 13^{3 k+6}(B 0)$,

and the result is still true for $k=-1$, so we have

(34) $04113^{2 k}(B 0) 0^{k} \vdash\left(3 k^{2}+8 k+8\right) \quad 13^{3 k+3}(B 0)$.

Using (28), (30), (5), (6), (13) and (29) we get

$04123^{2 k+2}(B 0) 0^{k+2} \vdash\left(3 k^{2}+20 k+30\right) \quad 13^{3 k+7}(B 0)$,

and the result is still true for $k=-1$, so we have

(35) $\quad 04123^{2 k}(B 0) 0^{k+1} \vdash\left(3 k^{2}+14 k+13\right) \quad 13^{3 k+4}(B 0)$.

Using (28), (14), (6), (13) and (29) we get

$0413^{2 k+2}(B 0) 0^{k+1} \vdash\left(3 k^{2}+14 k+17\right) \quad 13^{3 k+5}(B 0)$,

and the result is still true for $k=-1$, so we have

(36) $0413^{2 k}(B 0) 0^{k} \vdash\left(3 k^{2}+8 k+6\right) \quad 13^{3 k+2}(B 0)$.

Using (28), (30), (6), (13) and (29) we get

$$
0423^{2 k+2}(B 0) 0^{k+2} \vdash\left(3 k^{2}+20 k+28\right) \quad 13^{3 k+6}(B 0),
$$

and the result is still true for $k=-1$, so we have

(37) $0423^{2 k}(B 0) 0^{k+1} \vdash\left(3 k^{2}+14 k+11\right) \quad 13^{3 k+3}(B 0)$.

Using (28), (21) and (29) we get

$$
043^{2 k+2}(B 0) 0^{k+1} \vdash\left(3 k^{2}+14 k+15\right) \quad 13^{3 k+4}(B 0),
$$

and the result is still true for $k=-1$, so we have

$$
\text { (38) } 043^{2 k}(B 0) 0^{k} \vdash\left(3 k^{2}+8 k+4\right) \quad 13^{3 k+1}(B 0) .
$$

Using (28), (17), (24) and (11) we get

$$
13^{2 k+3}(B 0) 0^{k+1} \vdash\left(3 k^{2}+14 k+15\right) \quad 23^{3 k+4}(B 0),
$$

and the result is still true for $k=-1$, so we have

$$
\text { (39) } 13^{2 k+1}(B 0) 0^{k} \quad \vdash\left(3 k^{2}+8 k+4\right) \quad 23^{3 k+1}(B 0) .
$$

Using (28), (18), (24) and (11) we get

$$
23^{2 k+3}(B 0) 0^{k+1} \vdash\left(3 k^{2}+14 k+15\right) \quad 3^{3 k+5}(B 0),
$$

and the result is still true for $k=-1$, so we have

$$
\text { (40) } 23^{2 k+1}(B 0) 0^{k} \quad \vdash\left(3 k^{2}+8 k+4\right) \quad 3^{3 k+2}(B 0) .
$$

Using (28), (16), (2) and (29) we get

$$
0^{3} 3^{2 k+3}(B 0) 0^{k+1} \vdash\left(3 k^{2}+14 k+33\right) \quad 41^{2} 3^{3 k+4}(B 0),
$$

and the result is still true for $k=-1$, so we have

$$
\text { (41) } \quad 0^{3} 3^{2 k+1}(B 0) 0^{k} \quad \vdash\left(3 k^{2}+8 k+22\right) \quad 41^{2} 3^{3 k+1}(B 0) .
$$

Using (39) we get

(42) $04113^{2 k+1}(B 0) 0^{k} \quad \vdash\left(3 k^{2}+8 k+4\right) \quad 04123^{3 k+1}(B 0)$.

Using (40) we get

(43) $04123^{2 k+1}(B 0) 0^{k} \quad \vdash\left(3 k^{2}+8 k+4\right) \quad 0413^{3 k+2}(B 0)$. 
Using (39) we get

(44) $0413^{2 k+1}(B 0) 0^{k} \quad \vdash\left(3 k^{2}+8 k+4\right) \quad 0423^{3 k+1}(B 0)$.

Using (40) we get

(45) $0423^{2 k+1}(B 0) 0^{k} \vdash\left(3 k^{2}+8 k+4\right) \quad 043^{3 k+2}(B 0)$.

Using (28) and (20) we get

$43^{2 k+3}(B 0) 0^{k+1} \vdash\left(3 k^{2}+11 k+11\right) \quad 1(H 2) 2^{3 k+3} 0$,

and the result is still true for $k=-1$, so we have

(46) $43^{2 k+1}(B 0) 0^{k} \quad \vdash\left(3 k^{2}+5 k+3\right) \quad 1(H 2) 2^{3 k} 0$.

Results (1) and (31)-(46) give results (a)-(p) of the theorem.

Using the rules of this theorem, we have, in 2002 transitions,

$$
{ }^{\omega} 0(A 0) 0^{\omega} \vdash(1) \quad C(0,1) \vdash(4) \quad C(1,1) \vdash() \cdots \vdash() \quad \text { END. }
$$

Let $g_{3}$ be the pure Collatz-like function with parameter defined by: for $k \geq 0$,

$$
\begin{aligned}
& g_{3}(2 k, 1)=(3 k+1,1) \quad g_{3}(2 k+1,1)=(3 k+1,2) \\
& g_{3}(2 k, 2)=(3 k+2,1) \quad g_{3}(2 k+1,2)=(3 k+2,3) \\
& g_{3}(2 k, 3)=(3 k, 1) \quad g_{3}(2 k+1,3)=(3 k+1,4) \\
& g_{3}(2 k, 4)=(3 k+3,1) \quad g_{3}(2 k+1,4)=(3 k+1,5) \\
& g_{3}(2 k, 5)=(3 k+4,1) \quad g_{3}(2 k+1,5)=(3 k+2,6) \\
& g_{3}(2 k, 6)=(3 k+2,1) \quad g_{3}(2 k+1,6)=(3 k+1,7) \\
& g_{3}(2 k, 7)=(3 k+3,1) \quad g_{3}(2 k+1,7)=(3 k+2,8) \\
& g_{3}(2 k, 8)=(3 k+1,1) \quad g_{3}(2 k+1,8) \quad \text { undefined }
\end{aligned}
$$

Then $g_{3}^{2001}(0,1)$ is undefined.

Proposition 5.2. The behavior of Turing machine $M_{3}$, on inputs $02^{n}, n \geq 1$, depends on the behavior of iterated $g_{3}^{k}(n, 1), k \geq 1$.

Proof. We have ${ }^{\omega} 0(A 0) 2^{n} 0^{\omega} \vdash(n+1) \quad{ }^{\omega} 013^{n}(B 0) 0^{\omega}=C(n, 1)$.

Since the behavior of iterated $g_{3}^{k}(n, 1)$ is an open problem in mathematics, this si also the case for the halting problem for Turing machine $M_{3}$.

Note that the way by which a high score is obtained is particularly clear for machine $M_{3}$. The parameter $p, 1 \leq p \leq 8$, can be seen as a state. If $n$ is odd, $g_{3}(n, p)=\left(n^{\prime}, p+1\right)$, the state goes from $p$ to $p+1$, and the computation stops when state 8 is reached. If $n$ is even, $g_{3}(n, p)=\left(n^{\prime}, 1\right)$, and the state goes back to 1 .

\section{Exponential Collatz-like}

Let $M_{4}$ be the $6 \times 2$ Turing machine defined by Table 9 ,

We have $s\left(M_{4}\right)>7.4 \times 10^{36534}$ and $\sigma\left(M_{4}\right)>3.5 \times 10^{18267}$.

This machine is the current champion for the busy beaver competition for $6 \times 2$ machines. It was discovered in June 2010 by Pavel Kropitz.

The following theorem gives the rules observed by Turing machine $M_{4}$.

Theorem 6.1. We have the following transitions between configurations of Turing machine $M_{4}$. Let $C(n)={ }^{\omega} 0(A 0) 1^{n} 0^{\omega}$. Then 


\begin{tabular}{|c|c|c|}
\hline$M_{4}$ & 0 & 1 \\
\hline$A$ & $1 \mathrm{RB}$ & $1 \mathrm{LE}$ \\
\hline$B$ & $1 \mathrm{RC}$ & $1 \mathrm{RF}$ \\
\hline$C$ & $1 \mathrm{LD}$ & $0 \mathrm{RB}$ \\
\hline$D$ & $1 \mathrm{RE}$ & $0 \mathrm{LC}$ \\
\hline$E$ & $1 \mathrm{LA}$ & $0 \mathrm{RD}$ \\
\hline$F$ & $1 \mathrm{RH}$ & $1 \mathrm{RC}$ \\
\hline
\end{tabular}

Table 9: Machine $M_{4}$ discovered in June 2010 by P. Kropitz
(a) $C(0) \vdash(29) \quad C(9)$,
(b) $C(2) \vdash(36) \quad C(11)$,
(c) $C(3) \vdash(48) \quad C(13)$,

and, for all $k \geq 0$,

(d) $C(3 k+1) \quad \vdash(3 k+3)$

(e) $C(9 k+5) \quad \vdash\left(\left(4802 \times 16^{k+1}+6370 \times 4^{k+1}+2280 k-25362\right) / 270\right)$

(f) $C(9 k+6) \vdash\left(\left(125 \times 16^{k+2}-575 \times 4^{k+2}+228 k-2226\right) / 27\right)$

(g) $C(9 k+8) \quad \vdash\left(\left(4802 \times 16^{k+1}+6370 \times 4^{k+1}+2280 k-11592\right) / 270\right)$

${ }^{\omega} 0111(011)^{k}(H 0) 0^{\omega}$, $C\left(\left(98 \times 4^{k}-11\right) / 3\right)$, $C\left(\left(50 \times 4^{k+1}-59\right) / 3\right)$,

(h) $C(9 k+9) \vdash\left(\left(125 \times 16^{k+2}+325 \times 4^{k+2}+228 k-2289\right) / 27\right)$

(i) $C(9 k+11) \vdash\left(\left(4802 \times 16^{k+2}-11270 \times 4^{k+2}+2280 k-22452\right) / 270\right)$ $C\left(\left(98 \times 4^{k}+1\right) / 3\right)$,

(j) $C(9 k+12) \vdash\left(\left(125 \times 16^{k+2}+325 \times 4^{k+2}+228 k-912\right) / 27\right)$ $C\left(\left(50 \times 4^{k+1}-11\right) / 3\right)$ $C\left(\left(98 \times 4^{k+1}-59\right) / 3\right)$

Note that the behavior of this Turing machine on the blank tape involves only items (a), (d), (h) and (j).

Proof. A direct inspection of the transition table gives
(1) $0^{3}(A 0) 0^{6} \quad \vdash(29) \quad(A 0) 1^{9}$,
(2) $0^{4}(A 0) 1^{2} 0^{5} \quad \vdash(36) \quad(A 0) 1^{11}$,
(3) $0^{4}(A 0) 1^{3} 0^{6} \vdash(48) \quad(A 0) 1^{13}$,
(4) $(A 0) 1 \quad \vdash(1) \quad 1(B 1)$,
(5) $01(E 0) \quad \vdash(2) \quad(E 0) 11$,
(6) $0(E 0) \quad \vdash(1) \quad(A 0) 1$,
(7) $11(E 0) \quad \vdash(2) \quad(E 1) 11$,
(8) $01(C 0) \quad \vdash(2) \quad(C 0) 01$,
(9) $11(C 0) \quad \vdash(2) \quad(C 1) 01$,
(10) $0(C 0) \quad \vdash(2) \quad 1(E 1)$,
(11) $(E 1) 01 \quad \vdash(2) \quad 01(E 1)$,
$(12)(E 1) 00 \quad \vdash(2) \quad 01(E 0)$,

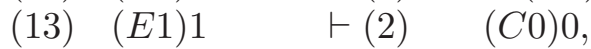
(14) $(B 1) 1^{3} \quad \vdash(3) \quad 110(B 1)$,
$(15)(B 1) 00 \quad \vdash(2) \quad 11(H 0)$,
(16) $(B 1) 10 \quad \vdash(6) \quad 01(C 1)$,
(17) $(B 1) 1100 \quad \vdash(12) \quad(01)^{2}(C 1)$,
(18) $(C 1) 01 \quad \vdash(2) \quad 01(C 1)$,
(19) $(C 1) 00 \quad \vdash(2) \quad 01(C 0)$,
(20) $(C 1) 1^{6} 0^{6} \quad \vdash(44) \quad 01(E 1) 1^{10}$,
(21) $(C 1) 1^{8} 0^{11} \vdash(113) \quad 1(01)^{5}(E 1) 1^{8}$.

Iterating, respectively, (5), (8), (11), (14) and (18) gives 
(22) $(01)^{k}(E 0) \quad \vdash(2 k) \quad(E 0) 1^{2 k}$,

(23) $(01)^{k}(C 0) \vdash(2 k) \quad(C 0)(01)^{k}$,

(24) $(E 1)(01)^{k} \vdash(2 k) \quad(01)^{k}(E 1)$,

$(25)(B 1) 1^{3 k} \vdash(3 k) \quad(110)^{k}(B 1)$,

(26) $(C 1)(01)^{k} \vdash(2 k) \quad(01)^{k}(C 1)$.

Using (19), (23), (10) and (24), we get

(27) $0(01)^{k}(C 1) 00 \quad \vdash(4 k+8) \quad 1(01)^{k+1}(E 1)$.

Using (12), (22) and (6), we get

(28) $0(01)^{k}(E 1) 00 \quad \vdash(2 k+5) \quad(A 0) 1^{2 k+3}$.

Using (12), (22) and (7), we get

(29) $11(01)^{k}(E 1) 00 \quad \vdash(2 k+6) \quad(E 1) 1^{2 k+4}$.

Using (13), (23), (10) and (24), we get

(30) $0(01)^{k}(E 1) 1 \quad \vdash(4 k+4) \quad 1(01)^{k}(E 1) 0$.

Using (13), (23), (9) and (26), we get

(31) $11(01)^{k}(E 1) 1 \quad \vdash(4 k+6) \quad(01)^{k+1}(C 1) 0$.

Using (20), (30), (11), (31) and (18), we get

(32) $10(01)^{k}(C 1) 1^{6} 0^{6} \vdash(8 k+70) \quad(01)^{k+4}(C 1) 1^{6}$.

By induction on $n$, using (32), we get

(33) $(10)^{n}(01)^{k}(C 1) 1^{6} 0^{6 n} \vdash\left(16 n^{2}+8 k n+54 n\right) \quad(01)^{4 n+k}(C 1) 1^{6}$.

Using (30) and (11), we get

(34) $00(01)^{k}(E 1) 11 \quad \vdash(4 k+6) \quad(01)^{k+2}(E 1)$.

By induction on $n$, using (34), we get

(35) $0^{2 n}(01)^{k}(E 1) 1^{2 n} \vdash\left(4 n^{2}+4 k n+2 n\right) \quad(01)^{2 n+k}(E 1)$.

Using (20), (31), (18), (21), (31), (18) and (33), we get

(36) $11(01)^{k}(C 1) 1^{6} 0^{6 k+29} \vdash\left(16 k^{2}+178 k+481\right) \quad 0(01)^{4 k+15}(C 1) 1^{6}$.

Using (20), (35) and (28), we get

(37) $0^{11}(01)^{k}(C 1) 1^{6} 0^{8} \quad \vdash(22 k+201) \quad(A 0) 1^{2 k+25}$.

Using (20), (35), (31), (18), (32) and (36), we get

(38) $(110)^{3} 0(01)^{k}(C 1) 1^{6} 0^{6 k+101} \vdash\left(16 k^{2}+514 k+4045\right) \quad 0(01)^{4 k+55}(C 1) 1^{6}$.

By induction on $k$, using (38) we get

(39) $(110)^{3 k} 0(01)^{n}(C 1) 1^{6} 0^{a} \vdash(T) \quad 0(01)^{b}(C 1) 1^{6}$,

with $a=\left(2(3 n+55) 4^{k}-6 n-27 k-110\right) / 3, b=\left((3 n+55) 4^{k}-55\right) / 3$, and $T=\frac{16(3 n+55)^{2}}{135} 16^{k}-$ $\frac{218(3 n+55)}{27} 4^{k}-\frac{5}{9} k-\frac{16(3 n+55)^{2}}{135}+\frac{218(3 n+55)}{27}$.

Using (4), (25), (17), (27), (29), (31), (18), (21), (31), (18) and (33), we get

(40) $\quad(A 0) 1^{3 k+9} 0^{29} \vdash(3 k+484) \quad 1(110)^{k} 0(01)^{15}(C 1) 1^{6}$.

Using (40), (39), (32) and (37), we get

(41) $0^{11}(A 0) 1^{9 k+9} 0^{a} \vdash(T) \quad(A 0) 1^{b}$,

with $a=\left(50 \times 4^{k+1}-11\right) / 3-9 k-20, b=\left(50 \times 4^{k+1}-11\right) / 3$, and $T=\left(125 \times 16^{k+2}+\right.$ $\left.325 \times 4^{k+2}+228 k-2289\right) / 27$.

Using (40), (39), (20), (35), (31), (18) and (37), we get

(42) $0^{12}(A 0) 1^{9 k+12} 0^{a} \vdash(T) \quad(A 0) 1^{b}$,

with $a=\left(50 \times 4^{k+1}+1\right) / 3-9 k-24, b=\left(50 \times 4^{k+1}+1\right) / 3$, and $T=\left(125 \times 16^{k+2}+325 \times\right.$ $\left.4^{k+2}+228 k-912\right) / 27$. 
Using (4), (25) and (15), we get

(43) $(A 0) 1^{3 k+1} 00 \quad \vdash(3 k+3) \quad 111(011)^{k}(H 0)$.

Results (1), (43), (41) and (42) are results (a), (d), (h) and (j) of the theorem. They are sufficient to analyze the behavior of the Turing machine on a blank tape. The following gives its behavior from configurations ${ }^{\omega} 0(A 0) 1^{n} 0^{\omega}$, where $n=9 k+m, m \in\{5,6,8,11\}$. Using (4), (25), (16), (27), (29), (31), (18), (32) and (36), we get (44) $(A 0) 1^{3 k+14} 0^{88} \vdash(3 k+3076) \quad 1(110)^{k} 0(01)^{47}(C 1) 1^{6}$.

Using (44), (39), (32) and (37), we get

$$
0^{11}(A 0) 1^{9 k+14} 0^{a} \vdash(T) \quad(A 0) 1^{b},
$$

with $b=\left(98 \times 4^{k+1}-11\right) / 3$ and $T=\left(4802 \times 16^{k+2}+6370 \times 4^{k+2}+2280(k+1)-25362\right) / 270$, and this result is still true for $k=-1$, since

$$
0^{11}(A 0) 1^{5} 0^{13} \vdash(285) \quad(A 0) 1^{29},
$$

so we get

(45) $\quad 0^{11}(A 0) 1^{9 k+5} 0^{a} \vdash(T) \quad(A 0) 1^{b}$,

with $b=\left(98 \times 4^{k}-11\right) / 3$ and $T=\left(4802 \times 16^{k+1}+6370 \times 4^{k+1}+2280 k-25362\right) / 270$.

Using (20), (35), (31), (18), (32), (36) and (37), we get

(46) $\quad 0^{10} 1(110)^{2} 0(01)^{k}(C 1) 1^{6} 0^{6 k+103} \vdash\left(16 k^{2}+570 k+4886\right) \quad(A 0) 1^{8 k+127}$.

Using (40), (39) and (46), we get

$$
0^{10}(A 0) 1^{9 k+15} 0^{a} \vdash(T) \quad(A 0) 1^{b},
$$

with $b=\left(50 \times 4^{k+2}-59\right) / 3$ and $T=\left(125 \times 16^{k+3}-575 \times 4^{k+3}+228(k+1)-2226\right) / 27$, and this result is still true for $k=-1$, since

$$
0^{10}(A 0) 1^{6} 0^{31} \vdash(762) \quad(A 0) 1^{47},
$$

so we get

$$
\text { (47) } \quad 0^{10}(A 0) 1^{9 k+6} 0^{a} \vdash(T) \quad(A 0) 1^{b},
$$

with $b=\left(50 \times 4^{k+1}-59\right) / 3$ and $T=\left(125 \times 16^{k+2}-575 \times 4^{k+2}+228 k-2226\right) / 27$.

Using (20), (30), (11), (31), (18) and (37), we get

$$
\text { (48) } \quad 0^{12} 11100(01)^{k}(C 1) 1^{6} 0^{14} \vdash(30 k+407) \quad(A 0) 1^{2 k+37} .
$$

Using (44), (39) and (48), we get

$$
0^{12}(A 0) 1^{9 k+17} 0^{a} \vdash(T)(A 0) 1^{b},
$$

with $b=\left(98 \times 4^{k+1}+1\right) / 3$ and $T=\left(4802 \times 16^{k+2}+6370 \times 4^{k+2}+2280(k+1)-11592\right) / 270$, and this result is still true for $k=-1$, since

so we get

$$
0^{12}(A 0) 1^{8} 0^{13} \vdash(336) \quad(A 0) 1^{33},
$$

(49) $0^{12}(A 0) 1^{9 k+8} 0^{a} \vdash(T) \quad(A 0) 1^{b}$,

with $b=\left(98 \times 4^{k}+1\right) / 3$ and $T=\left(4802 \times 16^{k+1}+6370 \times 4^{k+1}+2280 k-11592\right) / 270$.

Using (44), (39) and (46), we get

$$
0^{10}(A 0) 1^{9 k+20} 0^{a} \vdash(T) \quad(A 0) 1^{b},
$$

with $b=\left(98 \times 4^{k+2}-59\right) / 3$ and $T=\left(4802 \times 16^{k+3}-11270 \times 4^{k+3}+2280(k+1)-22452\right) / 270$, and this result is still true for $k=-1$, since

so we get

$$
0^{10}(A 0) 1^{11} 0^{90} \vdash(3802) \quad(A 0) 1^{111},
$$

(50) $0^{10}(A 0) 1^{9 k+11} 0^{a} \vdash(T) \quad(A 0) 1^{b}$, with $b=\left(98 \times 4^{k+1}-59\right) / 3$ and $T=\left(4802 \times 16^{k+2}-11270 \times 4^{k+2}+2280 k-22452\right) / 270$.

Results (45), (47), (49) and (50) are results (e), (f), (g) and (i) of the theorem. 
Results (1), (2) and (3) give special cases (a), (b) and (c) of the theorem.

Using the rules of this theorem, we have,

$$
\begin{gathered}
{ }^{\omega} 0(A 0) 0^{\omega} \vdash(29) \quad C(9) \vdash(1293) \quad C(63) \vdash(19,884,896,677) \\
C(273063) \vdash\left(T_{1}\right) \quad C\left(\left(50 \times 4^{30340}+1\right) / 3\right) \vdash\left(T_{2}\right) \quad{ }^{\omega} 0111(011)^{K}(H 0) 0^{\omega},
\end{gathered}
$$

with $T_{1}=\left(125 \times 16^{30341}+325 \times 4^{30341}+6916380\right) / 27, T_{2}=\left(50 \times 4^{30340}+7\right) / 3, K=$ $\left(50 \times 4^{30340}-2\right) / 9$.

The total time is $s\left(M_{4}\right)=\left(125 \times 16^{30341}+1750 \times 4^{30340}+15\right) / 27+19,885,154,163$, and the final number of symbols 1 is $\sigma\left(M_{4}\right)=\left(25 \times 4^{30341}+23\right) / 9$.

Let us give some informal comments on how Turing machine $M_{4}$ works. As in the case of machine $M_{1}$, edge effects give the multiplicity of cases. Also as in the case of machine $M_{1}$, machine $M_{4}$ nibbles strings $(01)^{n}$, transforming them into strings four times longer (see transitions (32) and (33) in the proof of Theorem 6.1). A new idea allows machine $M_{4}$ to reach exponential scores. Machine $M_{4}$ nibbles strings $(110)^{3 k}$ and, each time a string $(110)^{3}$ is nibbled, the length of a string $(01)^{n}$ becomes four times longer (see transitions (38) and (39) in the proof of Theorem 6.1). This explains why powers of four are obtained in the transitions of Theorem 6.1.

Let $g_{4}$ be the exponential Collatz-like function defined by: for $k \geq 0$,

$$
\begin{aligned}
g_{4}(0) & =9, \\
g_{4}(2) & =11, \\
g_{4}(3) & =13, \\
g_{4}(3 k+1) & \text { undefined, } \\
g_{4}(9 k+5) & =\left(98 \times 4^{k}-11\right) / 3, \\
g_{4}(9 k+6) & =\left(50 \times 4^{k+1}-59\right) / 3, \\
g_{4}(9 k+8) & =\left(98 \times 4^{k}+1\right) / 3, \\
g_{4}(9 k+9) & =\left(50 \times 4^{k+1}-11\right) / 3, \\
g_{4}(9 k+11) & =\left(98 \times 4^{k+1}-59\right) / 3, \\
g_{4}(9 k+12) & =\left(50 \times 4^{k+1}+1\right) / 3 .
\end{aligned}
$$

Then $g_{4}^{5}(0)$ is undefined.

The theorem gives immediately the following proposition.

Proposition 6.2. The behavior of Turing machine $M_{4}$, on inputs $01^{n}, n \geq 0$, depends on the behavior of iterated $g_{4}^{k}(n), k \geq 1$.

Since the behavior of iterated $g_{4}^{k}(n)$ is an open problem in mathematics, this is also the case for the halting problem for Turing machine $M_{4}$.

Let $h_{4}(n)=\min \left\{k: g_{4}^{k}(n)\right.$ is undefined $\}$. We have seen that $h_{4}(0)=5$. We also have $h_{4}(2)=8$, and $C(2) \vdash(T)$ END with $T>10^{10^{10^{10^{18641000}}}}$. We also have $h_{4}(36)=15$.

\section{UNCLASSIFIABLE MACHINE}

Let $M_{5}$ be the $6 \times 2$ Turing machine defined by Table 10 ,

We have $s\left(M_{5}\right)>3.8 \times 10^{21132}$ and $\sigma\left(M_{5}\right)>3.1 \times 10^{10566}$.

This machine was discovered in May 2010 by Pavel Kropitz. It was the champion for the busy beaver competition for $6 \times 2$ machines from May to June 2010 . 


\begin{tabular}{|c|c|c|}
\hline$M_{5}$ & 0 & 1 \\
\hline$A$ & $1 \mathrm{RB}$ & $0 \mathrm{LD}$ \\
\hline$B$ & $1 \mathrm{RC}$ & $0 \mathrm{RF}$ \\
\hline$C$ & $1 \mathrm{LC}$ & $1 \mathrm{LA}$ \\
\hline$D$ & $0 \mathrm{LE}$ & $1 \mathrm{RH}$ \\
\hline$E$ & $1 \mathrm{LA}$ & $0 \mathrm{RB}$ \\
\hline$F$ & $0 \mathrm{RC}$ & $0 \mathrm{RE}$ \\
\hline
\end{tabular}

Table 10: Machine $M_{5}$ discovered in May 2010 by P. Kropitz

The following theorem is adapted from an analysis of S. Ligocki [Li10]. It gives the rules that enable Turing machine $M_{5}$ to reach a halting configuration from a blank tape.

Theorem 7.1. We have the following transitions between configurations of Turing machine $M_{5}$. Let $C(k, n)={ }^{\omega} 010^{n} 1(C 1) 1^{3 k} 0^{\omega}$. Then

(a) ${ }^{\omega} 0(A 0) 0^{\omega} \quad \vdash(47) \quad C(2,5)$,

and, for all $k \geq 0$,
(b) $C(k, 0)$
$\vdash(3)$
${ }^{\omega} 01(H 0) 1^{3 k+1} 0^{\omega}$,
(c) $C(k, 1)$
$\vdash(3 k+37)$
$C(2,3 k+2)$,
(d) $C(k, 2)$
$\vdash(12 k+44)$
$C(k+2,4)$,
(e) $C(k, 3) \quad \vdash(3 k+57)$
$C(2,3 k+8)$,
(f) $C(k, n+4) \quad \vdash\left(27 k^{2}+105 k+112\right)$
$C(3 k+5, n)$.

Proof. A direct inspection of the transition table gives
(1) $\quad 0^{4}(A 0) 0^{9}$
$\vdash(47) \quad 10^{5} 1(C 1) 1^{6}$,
(2) $0(C 0)$
$\vdash(1) \quad(C 0) 1$,
(3) $1(C 0)$
$\vdash(1) \quad(C 1) 1$,
(4) $\quad(B 1) 00$
$\vdash(2) \quad 0^{2}(C 0)$,
(5) $\quad(B 1) 01$
$\vdash(4) \quad 01(B 1)$,
(6) $\quad(B 1) 10$
$\vdash(4) \quad 01(B 1)$,
(7) $\quad(B 1) 10^{3}$
$\vdash(14) \quad 10^{3}(B 1)$,
(8) $(B 1) 1^{2} 0^{2} \quad \vdash(7) \quad 0^{2} 1(B 1) 1$,
$\begin{array}{lll}(9) \quad(B 1) 1^{3} & \vdash(3) & 0^{3}(B 1) \text {, }\end{array}$
(10) $0(C 1) \quad \vdash(2) \quad 1(B 1)$,
(11) $11(C 1) \quad \vdash(3) \quad 1(H 0) 1$,
(12) $0^{3} 1(C 1) \quad \vdash(10) \quad(C 1) 1^{4}$,
(13) $0^{3} 10^{2} 1(C 1) \quad \vdash(8) \quad 1(B 1) 0^{2} 10^{2} 1$,
(14) $101(C 1) \quad \vdash(8) \quad 1(B 1) 1^{2}$.

Iterating, respectively, (2) and (9) gives

(15) $0^{k}(C 0) \quad \vdash(k) \quad(C 0) 1^{k}$,

(16) $\quad(B 1) 1^{3 k} \vdash(3 k) \quad 0^{3 k}(B 1)$.

Using (4), (15) and (3), we get

(17) $10^{k}(B 1) 00 \vdash(k+5) \quad(C 1) 1^{k+3}$.

Using (16), (17) and (10), we get

(18) $01(B 1) 1^{3 k} 00 \quad \vdash(6 k+7) \quad 1(B 1) 1^{3 k+3}$.

By induction on $k$, using (18), we get

(19) $0^{k} 1(B 1) 0^{2 k} \vdash\left(3 k^{2}+4 k\right) \quad 1(B 1) 1^{3 k}$. 
Using (12), (10), (16), (6), (19), (16) and (17), we get

(20) $0^{4} 1(C 1) 1^{3 k} 0^{6 k+11} \vdash\left(27 k^{2}+105 k+112\right) \quad 1(C 1) 1^{9 k+15}$.

Using (14), (16), (8), (7) and (17), we get

(21) $101(C 1) 1^{3 k} 0^{7} \vdash(3 k+37) \quad 10^{3 k+2} 1(C 1) 1^{6}$.

Using (13), (17), (10), (9), (6), (5), (16), (17), (10), (16) and (17), we get

(22) $\quad 0^{4} 10^{2} 1(C 1) 1^{3 k} 0^{4} \vdash(12 k+44) \quad 10^{4} 1(C 1) 1^{3 k+6}$.

Using (12), (12), (10), (16), (8), (6), (17), (10), (9) and (17), we get

(23) $0^{4} 10^{3} 1(C 1) 1^{3 k} 0^{7} \vdash(3 k+57) \quad 10^{3 k+8} 1(C 1) 1^{6}$.

Results (1), (11), (21), (22), (23) and (20) give results (a)-(f) of the theorem.

Using the rules of this theorem, we have, in 22158 transitions,

$$
{ }^{\omega} 0(A 0) 0^{\omega} \vdash(47) \quad C(2,5) \vdash(430) \quad C(11,1) \vdash() \cdots \vdash() \quad \text { END. }
$$

Let us give some informal comments on how Turing machine $M_{5}$ works. The computation of machine $M_{5}$ on a blank tape is a succession of two types of phases. In the first one, the second parameter $p$ of configuration $C(n, p)$ is smaller than four, and transitions (b)-(e) of Theorem 7.1 immediately give a greater value to this second parameter. In the second type of phase, the second parameter is greater than four, and slowly decreases, while the first parameter rapidly increases. In this second type of phase, machine $M_{5}$ nibbles strings of symbols 0 two by two, and each time a string of two symbols 0 is nibbled, a string of three symbols 1 is produced (see transitions (18) and (19) in the proof of Theorem [7.1). In the computation of Turing machine $M_{5}$ on a blank tape, phases of the second type occur four times:

- from $C(2,5)$ to $C(11,1)$ (1 transition),

- from $C(2,35)$ to $C(29522,3)$ (8 transitions),

- from $C(2,88574)$ to $C(m, 2)$ (22143 transitions),

- from $C(m+2,4)$ to $C(3(m+2)+5,0)(1$ transition $)$,

where $m$ is a big number.

Let $g_{5}$ be the partial function defined by: for $k, n \geq 0$,

$$
\begin{aligned}
g_{5}(k, 0) & \text { undefined, } \\
g_{5}(k, 1) & =(2,3 k+2), \\
g_{5}(k, 2) & =(k+2,4), \\
g_{5}(k, 3) & =(2,3 k+8), \\
g_{5}(k, n+4) & =(3 k+5, n) .
\end{aligned}
$$

Then $g_{5}^{22157}(2,5)$ is undefined.

Proposition 7.2. The behavior of Turing machine $M_{5}$, on inputs $01^{3 n+3}, n \geq 0$, depends on the behavior of iterated $g_{5}^{k}(2,3 n+2)$.

Proof. We have ${ }^{\omega} 0(A 0) 1^{3 n+3} 0^{\omega} \vdash(3 n+30) \quad{ }^{\omega} 01^{3 n+2} 1(C 1) 1^{6} 0^{\omega}=C(2,3 n+2)$.

Since the behavior of iterated $g_{5}^{k}(n, p)$ is an open problem in mathematics, this is also the case for the halting problem for Turing machine $M_{5}$.

The following proposition shows that some configurations take a long time to halt.

Proposition 7.3. For Turing machine $M_{5}$, we have $C(9,1) \vdash(T)$ END with $T>$

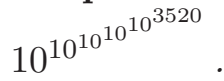


Proof. By induction on $n$, using Theorem 7.1 (f), it is easy to prove that, if $n \geq 0,0 \leq r \leq 3$, we have

$$
C(2,4 n+r) \vdash\left(t_{n}\right) \quad C\left(u_{n}, r\right),
$$

with $u_{n}=\left(3^{n+2}-5\right) / 2$ and $t_{n}=\left(3 \times 9^{n+3}-80 \times 3^{n+3}+584 n-27\right) / 32$.

By induction on $k$, it is easy to prove that, if $k \geq 2$, we have

$$
3^{2^{k-1}} \equiv 2^{k+1}+1\left(\bmod 2^{k+2}\right)
$$

so the multiplicative order of 3 modulo $2^{k+2}$ is $2^{k}$ for $k \geq 1$. Thus we can prove that, for $k \geq 1, n, m \geq 0$, we have

$$
n \equiv m\left(\bmod 2^{k}\right) \Longleftrightarrow u_{n} \equiv u_{m}\left(\bmod 2^{k+1}\right) .
$$

Now, suppose that, for $a \in\{1,3\}, n, n^{\prime} \geq 1, q, q^{\prime} \geq 1,0 \leq r, r^{\prime} \leq 3$, we have

$$
C(n, a) \vdash(3 n+27+10 a) \quad C(2,3 n+3 a-1)=C(2,4 q+r) \vdash\left(t_{q}\right) \quad C\left(u_{q}, r\right),
$$

and

$$
C\left(n^{\prime}, a\right) \vdash\left(3 n^{\prime}+27+10 a\right) \quad C\left(2,3 n^{\prime}+3 a-1\right)=C\left(2,4 q^{\prime}+r^{\prime}\right) \vdash\left(t_{q^{\prime}}\right) \quad C\left(u_{q^{\prime}}, r^{\prime}\right),
$$

and let $k \geq 2$ such that $n \equiv n^{\prime}\left(\bmod 2^{k+1}\right)$. Then it is easy to prove that $r=r^{\prime}$ and $u_{q} \equiv u_{q^{\prime}}\left(\bmod 2^{k}\right)$. So the behavior of configurations $C(n, a)$ is mirrored by the behavior of configurations $C\left(n^{\prime}, a\right)$ with $n^{\prime} \leq 2^{k}$ for suitable $k$.

In the following computation on $C(9,1)$ :

$$
\begin{array}{llll}
C(9,1) & \vdash() & C(2,4 \times 7+1) & \vdash\left(t_{7}\right) \\
C(9839,1) & \vdash() & C(2,4 \times 7379+3) & \vdash\left(t_{7379}\right) \\
C\left(u_{7379}, 3\right) & \vdash() & C\left(2,4 \times q_{3}+r_{3}\right) & \vdash\left(t_{q_{3}}\right) \\
C\left(u_{q_{3}}, r_{3}\right) & \vdash() & C\left(2,4 \times q_{4}+r_{4}\right) & \vdash\left(t_{q_{4}}\right) \\
C\left(u_{q_{4}}, r_{4}\right) & \vdash() & C\left(2,4 \times q_{5}+r_{5}\right) & \vdash\left(t_{q_{5}}\right) \\
C\left(u_{q_{5}}, r_{5}\right) & \vdash() & C\left(2,4 \times q_{6}+r_{6}\right) & \vdash\left(t_{q_{6}}\right) \\
C\left(u_{q_{6}}, r_{6}\right) & \vdash(3) & \mathrm{END} &
\end{array}
$$

we know that $r_{6}=0$ because we have

$$
\begin{array}{llll}
u_{q_{1}}=u_{7} \equiv 47(\bmod 64), & (3 \times 47)+2=(4 \times 35)+3, & q_{2}^{\prime}=35, & r_{2}=3, \\
u_{q_{2}} \equiv u_{q_{2}^{\prime}} \equiv 23(\bmod 32), & (3 \times 23)+8=(4 \times 19)+1, & q_{3}^{\prime}=19, & r_{3}=1, \\
u_{q_{3}} \equiv u_{q_{3}^{\prime}} \equiv 7(\bmod 16), & (3 \times 7)+2=(4 \times 5)+3, & q_{4}^{\prime}=5, & r_{4}=3, \\
u_{q_{4}} \equiv u_{q_{4}^{\prime}} \equiv 3(\bmod 8), & (3 \times 3)+8=(4 \times 4)+1, & q_{5}^{\prime}=4, & r_{5}=1, \\
u_{q_{5}} \equiv u_{q_{5}^{\prime}} \equiv 2(\bmod 4), & (3 \times 2)+2=(4 \times 2)+0, & q_{6}^{\prime}=2, & r_{6}=0 .
\end{array}
$$

It is easy to see that, if $a \in\{1,3\}, n \geq 0$, and

$$
C(n, a) \vdash(3 n+27+10 a) \quad C(2,3 n+3 a-1)=C(2,4 q+r) \vdash\left(t_{q}\right) \quad C\left(u_{q}, r\right),
$$

then $q \geq(3 n-1) / 4$ and $u_{q}>\left(3^{3 / 4}\right)^{n}>2^{n}$.

And we also have $n \geq 5 \Rightarrow t_{n}>68 \times 9^{n}$, so, if $C(9,1) \vdash(T)$ END, we have

$$
T>t_{q_{6}}>9^{q_{6}}>9^{3 u_{q_{5}} / 4}>5^{u_{q_{5}}}
$$

and $u_{q_{5}}>2^{u_{q_{4}}}, u_{q_{4}}>2^{u_{q_{3}}}, u_{q_{3}}>2^{u_{q_{2}}}=2^{u_{7379}}$, so $T>5^{2^{2^{2^{u_{73}}}}}$.

Using $u_{7379}>10^{3521}$, and, for $x \geq 1,2^{10^{x}}>10^{10^{x-.53}}, 2^{10^{10^{x}}}>10^{10^{10^{x-.03}}}, 2^{10^{10^{10^{x}}}}>$ $10^{10^{10^{10^{x-.03}}}}$ and $5^{10^{10^{10^{10^{x}}}}}>10^{10^{10^{10^{10^{x-}}}}}$, we are done. 


\begin{tabular}{|c|c|c|}
\hline$M_{6}$ & 0 & 1 \\
\hline$A$ & $1 \mathrm{RB}$ & $0 \mathrm{RF}$ \\
\hline$B$ & $0 \mathrm{LB}$ & $1 \mathrm{LC}$ \\
\hline$C$ & $1 \mathrm{LD}$ & $0 \mathrm{RC}$ \\
\hline$D$ & $1 \mathrm{LE}$ & $1 \mathrm{RH}$ \\
\hline$E$ & $1 \mathrm{LF}$ & $0 \mathrm{LD}$ \\
\hline$F$ & $1 \mathrm{RA}$ & $0 \mathrm{LE}$ \\
\hline
\end{tabular}

Table 11: Machine $M_{6}$ discovered in November 2007 by T. and S. Ligocki

\section{A Potentially infinite SET OF RULES}

Let $M_{6}$ be the $6 \times 2$ Turing machine defined by Table 11 .

We have $s\left(M_{6}\right)>8.9 \times 10^{1762}$ and $\sigma\left(M_{6}\right)>2.5 \times 10^{881}$.

This machine was discovered in November 2007 by Terry and Shawn Ligocki. It was the champion for the busy beaver competition for $6 \times 2$ machines from November to December 2007.

The complete analysis of Turing machine $M_{6}$ seems to need an infinite set of rules, but proving this assertion could be difficult. Of course, only a finite subset of these rules are needed when the machine is launched on a blank tape.

The following theorem gives the rules that enable Turing machine $M_{6}$ to reach a halting configuration from a blank tape.

Recall that $\operatorname{bin}(p)$ is the usual binary representation of number $p$, and $\mathrm{R}\left(w_{1} \ldots w_{n}\right)=$ $w_{n} \ldots w_{1}$.

Theorem 8.1. We have the following transitions between configurations of Turing machine $M_{6}$. Let $C(n, p)={ }^{\omega} 0(F 0)(10)^{n} R(b i n(p)) 0^{\omega}$, so that $C(k, 4 m+1)=C(k+1, m)$. Then

(a) ${ }^{\omega} 0(A 0) 0^{\omega} \quad \vdash(6) \quad C(0,15)$,

and, for all $k, m \geq 0$,
(b) $C(k, 4 m+3)$
$\vdash(4 k+6)$
$C(k+2, m)$,
(c) $C(2 k, 4 m)$
$\vdash\left(30 k^{2}+20 k+15\right)$
$\vdash\left(30 k^{2}+40 k+25\right)$
(d) $C(2 k+1,4 m)$
$\vdash(8 k+20)$
$C(5 k+2,2 m+1)$,
(e) $C(k, 8 m+2)$
$\vdash\left(30 k^{2}+40 k+23\right)$
$C(5 k+2,32 m+20)$,
(f) $C(2 k, 16 m+6)$
$\vdash\left(30 k^{2}+80 k+63\right)$
$C(k+3,2 m+1)$,
(g) $C(2 k+1,16 m+6)$
$\vdash(4 k+18)$
$C(5 k+2,32 m+20)$,
(h) $C(k, 32 m+14)$
$\vdash\left(30 k^{2}+40 k+39\right)$
$C(5 k+7,2 m+1)$,
(i) $C(2 k, 128 m+94)$
$\vdash\left(30 k^{2}+80 k+79\right)$
$C(k+3,2 m+1)$,
(j) $C(2 k+1,128 m+94)$
$\vdash(4 k+34)$
$C(5 k+2,256 m+84)$,
(k) $C(k, 256 m+190)$
$\vdash(2 k+43)$
$C(5 k+9, m)$
(l) $C(k, 512 m+30)$
$C(k+5, m)$, ${ }^{\omega} 0(10)^{k} 1(H 0)(10)^{2}(01)^{2} R(\operatorname{bin}(m)) 0^{\omega}$.

Proof. A direct inspection of the transition table gives 


$$
\begin{array}{llll}
(1) & 0^{4}(A 0) 0 & \vdash(6) & (F 0) 1^{4} 0, \\
(2) & 0(F 0) 00 & \vdash(9) & (F 0) 1^{3}, \\
(3) & (F 0) 10 & \vdash(2) & 10(F 0), \\
(4) & (F 0) 11 & \vdash(4) & (F 1) 10, \\
(5) & (F 0) 01 & \vdash(4) & 10(C 1), \\
(6) & 10(E 1) & \vdash(2) & (E 1) 10, \\
(7) & 1(E 1) & \vdash(2) & 1(H 0), \\
(8) & 0^{3}(E 1) & \vdash(3) & (F 0) 110, \\
(9) & 00(F 1) & \vdash(2) & (F 0) 10, \\
(10) & 10(F 1) & \vdash(2) & (F 1) 10, \\
(11) & 1(F 1) & \vdash(1) & (E 1) 0, \\
(12) & (C 1) 1 & \vdash(1) & 0(C 1), \\
(13) & (C 1) 0 & \vdash(1) & 0(C 0), \\
(14) & 0^{3}(C 0) & \vdash(3) & (F 0) 1^{3}, \\
(15) & 100(C 0) & \vdash(3) & (F 1) 1^{3} .
\end{array}
$$

Iterating, respectively, (3), (10), (6) and (12) gives

(16) $(F 0)(10)^{k} \vdash(2 k) \quad(10)^{k}(F 0)$,

(17) $(10)^{k}(F 1) \quad \vdash(2 k) \quad(F 1)(10)^{k}$,

(18) $(10)^{k}(E 1) \vdash(2 k) \quad(E 1)(10)^{k}$,

(19) $(C 1) 1^{k} \quad \vdash(k) \quad 0^{k}(C 1)$.

Using (5), (19) and (13), we get

(20) $(F 0) 01^{k+1} 0 \quad \vdash(k+5) \quad 10^{k+2}(C 0)$.

Using (16), (4), (17) and (9), we get

(21) $0^{2}(F 0)(10)^{k} 11 \quad \vdash(4 k+6) \quad(F 0)(10)^{k+2}$.

Using (16), (20), (15), (17), (9) and (21), we get

$(22) \quad 0^{4}(F 0)(10)^{k} 010 \vdash(8 k+20) \quad(F 0)(10)^{k+3} 1$.

Using (22) and (21), we get

(23) $0^{6}(F 0)(10)^{k}(01)^{2} \vdash(12 k+38) \quad(F 0)(10)^{k+5}$.

By induction on $n$, using (23), we get

(24) $0^{6 n}(F 0)(10)^{k}(01)^{2 n} \vdash\left(30 n^{2}+12 k n+8 n\right) \quad(F 0)(10)^{5 n+k}$.

Using (24), with $k=2$, we get

$(25) \quad 0^{6 k}(F 0)(10)^{2}(01)^{2 k} \vdash\left(30 k^{2}+32 k\right)(F 0)(10)^{5 k+2}$.

Using (16), (2), (4), (11), (18), (8) and (21), we get

(26) $\quad 0^{5}(F 0)(10)^{k+1} 0^{2} \quad \vdash(4 k+25) \quad(F 0)(10)^{2}(01)^{k} 00101$.

Using (26) and (25), we get

(27) $0^{6 k+5}(F 0)(10)^{2 k+1} 0^{2} \quad \vdash\left(30 k^{2}+40 k+25\right) \quad(F 0)(10)^{5 k+2} 00101$.

Using (25) and (22), we get

$(28) \quad 0^{6 k+4}(F 0)(10)^{2}(01)^{2 k+1} 0 \quad \vdash\left(30 k^{2}+72 k+36\right) \quad(F 0)(10)^{5 k+5} 1$.

Using (26) and (28), we get

$$
0^{6 k+9}(F 0)(10)^{2 k+2} 0^{2} \vdash\left(30 k^{2}+80 k+65\right) \quad(F 0)(10)^{5 k+7} 1,
$$

and the result is still true for $k=-1$, so we have

$(29) \quad 0^{6 k+3}(F 0)(10)^{2 k} 0^{2} \vdash\left(30 k^{2}+20 k+15\right) \quad(F 0)(10)^{5 k+2} 1$.

Using (16), (20), (14), (4), (11), (18), (8) and (21), we get (30) $\quad 0^{5}(F 0)(10)^{k} 01^{2} 0 \quad \vdash(4 k+23) \quad(F 0)(10)^{2}(01)^{k} 00101$. 
Using (30) and (25), we get

(31) $0^{6 k+5}(F 0)(10)^{2 k} 01^{2} 0 \quad \vdash\left(30 k^{2}+40 k+23\right) \quad(F 0)(10)^{5 k+2} 00101$.

Using (30) and (28), we get

$(32) \quad 0^{6 k+9}(F 0)(10)^{2 k+1} 01^{2} 0 \quad \vdash\left(30 k^{2}+80 k+63\right) \quad(F 0)(10)^{5 k+7} 1$.

Using (16), (20), (14), (4), (17) and (9), we get

(33) $0^{2}(F 0)(10)^{k} 01^{3} 0 \quad \vdash(4 k+18) \quad(F 0)(10)^{k+3} 1$.

Using (16), (20), (14), (4), (9) and (16), we get

(34) $(F 0)(10)^{k} 01^{4} 0 \quad \vdash(2 k+21) \quad(10)^{k} 1(10)^{2}(F 0) 1$.

Using (34), (4), (17), (11), (18), (8) and (21), we get

(35) $\quad 0^{5}(F 0)(10)^{k} 01^{4} 01 \quad \vdash(4 k+39) \quad(F 0)(10)^{2}(01)^{k} 00(10)^{3}$.

Using (35) and (25), we get

(36) $0^{6 k+5}(F 0)(10)^{2 k} 01^{4} 01 \quad \vdash\left(30 k^{2}+40 k+39\right) \quad(F 0)(10)^{5 k+2} 0^{2}(10)^{3}$.

Using (35), (25) and (22), we get

(37) $0^{6 k+9}(F 0)(10)^{2 k+1} 01^{4} 01 \quad \vdash\left(30 k^{2}+80 k+79\right) \quad(F 0)(10)^{5 k+9}$.

Using (16), (20), (14), (4), (9) and (16), we get

(38) $(F 0)(01)^{k} 01^{5} 0 \quad \vdash(2 k+22) \quad(10)^{k+3}(F 0) 1$.

Using (38), (4), (17) and (9), we get

(39) $0^{2}(F 0)(10)^{k} 01^{5} 01 \quad \vdash(4 k+34) \quad(F 0)(10)^{k+5}$.

Using (34), (3), (2), (4), (11), (18) and (7), we get

(40) $(F 0)(10)^{k} 01^{4} 0^{4} \vdash(2 k+43) \quad(10)^{k} 1(H 0)(10)^{2}(01)^{2}$.

Results (1), (21), (29), (27), (22), (31), (32), (33), (36), (37), (39) and (40) give results (a) $-(1)$ of the theorem.

Note that the rules (a)-(l) are written in their order of occurrence in the computation of Turing machine $M_{6}$ on the blank tape.

Using the rules of this theorem, we have, in 3346 transitions,

$$
{ }^{\omega} 0(A 0) 0^{\omega} \vdash(6) \quad C(0,15) \vdash(6) \quad C(2,3) \vdash() \cdots \vdash() \quad \text { END }
$$

We have

$$
\begin{aligned}
& { }^{\omega} 0(A 0) 0 \mathrm{R}(\operatorname{bin}(p)) 0^{\omega} \vdash(6) \quad{ }^{\omega} 0(F 0) 1^{4} 0 \mathrm{R}(\operatorname{bin}(p)) 0^{\omega} \\
= & C(0,32 p+15) \vdash(6) \quad C(2,8 p+3) \vdash(14) \quad C(4,2 p),
\end{aligned}
$$

so the behavior of Turing machine $M_{6}$ on inputs $00 x, x \in\{0,1\}^{*}$, depends on the behavior of configurations $C(n, p)$, and the halting problem for Turing machine $M_{6}$ depends on this behavior.

Let us give some informal comments on how Turing machine $M_{6}$ works. First, as in the case of the previous machines, machine $M_{6}$ nibbles strings $(01)^{2 n}$ and transforms them into strings $(01)^{5 n}$ (see transitions (23) and (24) in the proof of Theorem 8.1). This explains why some transitions of Theorem 8.1 looks like a Collatz-like behavior of type $2 \rightarrow 5$. Second, unlike the previous machines, edge effects are more chaotic. A study of Turing machine $M_{6}$ beyond the rules stated in Theorem 8.1 seems to lead to an infinite set of rules, but we could not prove this assertion. 


\begin{tabular}{|c|c|c|}
\hline$M_{7}$ & 0 & 1 \\
\hline$A$ & $1 \mathrm{RB}$ & $0 \mathrm{LB}$ \\
\hline$B$ & $0 \mathrm{RC}$ & $1 \mathrm{LB}$ \\
\hline$C$ & $1 \mathrm{RD}$ & $0 \mathrm{LA}$ \\
\hline$D$ & $1 \mathrm{LE}$ & $1 \mathrm{LF}$ \\
\hline$E$ & $1 \mathrm{LA}$ & $0 \mathrm{LD}$ \\
\hline$F$ & $1 \mathrm{RH}$ & $1 \mathrm{LE}$ \\
\hline
\end{tabular}

Table 12: Machine $M_{7}$ discovered in October 2000 by Marxen and Buntrock

\section{Configurations provably Stopping}

Let $M_{7}$ be the $6 \times 2$ Turing machine defined by Table 12

We have $s\left(M_{7}\right)>6.1 \times 10^{925}$ and $\sigma\left(M_{7}\right)>6.4 \times 10^{462}$.

This machine was discovered in October 2000 by Heiner Marxen and Jürgen Buntrock. It was the champion for the busy beaver competition for $6 \times 2$ machines from October 2000 to March 2001.

The following theorem was initially obtained by Munafo $\mathrm{MuWb}$. It gives the rules observed by Turing machine $M_{7}$.

Theorem 9.1. We have the following transitions between configurations of Turing machine $M_{7}$. Let $C(n)={ }^{\omega} 01^{n}(B 0) 0^{\omega}$. Then

(a) ${ }^{\omega} 0(A 0) 0^{\omega} \quad \vdash(1) \quad C(1)$,

and, for all $k \geq 0$,

(b) $C(3 k) \quad \vdash\left(54 \times 4^{k+1}-27 \times 2^{k+3}+26 k+86\right)$

(c) $C(3 k+1) \vdash\left(2048 \times\left(4^{k}-1\right) / 3-3 \times 2^{k+7}+26 k+792\right)$

$C\left(9 \times 2^{k+1}-8\right)$,

(d) $C(3 k+2) \vdash(3 k+8)$ $C\left(2^{k+5}-8\right)$, ${ }^{\omega} 01(H 1)(011)^{k} 01010^{\omega}$.

Proof. A direct inspection of the transition table gives

(1) $11(B 0) 00 \vdash(6) \quad(D 1) 0101$,

(2) $00(B 0) 01 \quad \vdash(8) \quad(B 0) 1^{4}$,

(3) $11(B 0) 01 \quad(6) \quad(B 1) 01^{3}$,

(4) $(B 0) 1 \quad \vdash(3) \quad 1(B 0)$,

(5) $0(B 1) \quad \vdash(1) \quad(B 0) 1$,

(6) $1(B 1) \quad \vdash(1) \quad(B 1) 1$,

(7) $0(D 1) \quad \vdash(2) \quad 1(H 1)$,

(8) $0^{3} 1(D 1) \quad \vdash(6) \quad(B 0) 1^{4}$,

(9) $0^{4} 1^{2}(D 1) \vdash(8) \quad(B 0) 1^{3} 01^{2}$,

(10) $1^{3}(D 1) \quad \vdash(3) \quad(D 1) 011$.

Iterating, respectively, (4), (6) and (10) gives

(11) $(B 0) 1^{k} \vdash(3 k) \quad 1^{k}(B 0)$,

(12) $1^{k}(B 1) \quad \vdash(k) \quad(B 1) 1^{k}$,

(13) $1^{3 k}(D 1) \vdash(3 k) \quad(D 1)(011)^{k}$.

Using (3), (12), (5) and (11), we get

$01^{k+2}(B 0) 01 \quad \vdash(4 k+10) \quad 1^{k+1}(B 0) 01^{3}$,

and the result is still true for $k=-1$, so we have

(14) $01^{k+1}(B 0) 01 \vdash(4 k+6) \quad 1^{k}(B 0) 01^{3}$.

By induction on $k$, using (14), we get 
(15) $0^{k} 1^{k}(B 0) 01 \quad \vdash\left(2 k^{2}+4 k\right) \quad(B 0) 01^{2 k+1}$.

Using (1), (13) and (7), we get

(16) $01^{3 k+2}(B 0) 0^{2} \quad \vdash(3 k+8) \quad 1(H 1)(011)^{k} 0101$.

Using (1), (13) and (9), we get

$0^{4} 1^{3 k+4}(B 0) 0^{2} \vdash(3 k+14) \quad(B 0) 1^{3}(011)^{k+1} 0101$,

and the result is still true for $k=-1$, so we have

(17) $0^{4} 1^{3 k+1}(B 0) 0^{2} \quad \vdash(3 k+11) \quad(B 0) 1^{3}(011)^{k} 0101$.

Using (1), (13) and (8), we get

(18) $0^{3} 1^{3 k+3}(B 0) 0^{2} \quad \vdash(3 k+12) \quad(B 0) 1^{4}(011)^{k} 0101$.

Using (11), (15) and (2), we get

(19) $\quad 0^{k+2}(B 0) 1^{k} 01 \quad \vdash\left(2 k^{2}+7 k+8\right) \quad(B 0) 1^{2 k+4}$.

By induction on $k$, using (19), we get

(20) $0^{2^{k}(n+5)-5-n-3 k}(B 0) 1^{n}(011)^{k} \vdash(T) \quad(B 0) 1^{2^{k}(n+5)-5}$,

with $T=2(n+5)^{2}\left(4^{k}-1\right) / 3-13(n+5)\left(2^{k}-1\right)+23 k$.

Using (20), for $n=3$ and $n=4$, we get respectively

(21) $0^{2^{k+3}-3 k-8}(B 0) 1^{3}(011)^{k} \quad \vdash\left(128\left(4^{k}-1\right) / 3-13 \times 2^{k+3}+23 k+104\right)$

(22) $0^{9 \times 2^{k}-3 k-9}(B 0) 1^{4}(011)^{k} \vdash\left(54 \times 4^{k}-117 \times 2^{k}+23 k+63\right)$

$(B 0) 1^{2^{k+3}-5}$

(B0) $1^{9 \times 2^{k}-5}$.

Using (11), (15), (2), (11), (15), (2) and (11), we get

(23) $0^{3 k+8}(B 0) 1^{k} 0101 \vdash\left(10 k^{2}+65 k+112\right) 1^{4 k+12}(B 0)$.

Using (17), (21) and (23), we get

(24) $0^{2^{k+5}-3 k-11} 1^{3 k+1}(B 0) 0^{2} \quad \vdash\left(2048 \times\left(4^{k}-1\right) / 3-3 \times 2^{k+7}+26 k+792\right) \quad 1^{2^{k+5}-8}(B 0)$.

Using (18), (22) and (23), we get

$$
0^{9 \times 2^{k+2}-3 k-13} 1^{3 k+3}(B 0) 0^{2} \quad \vdash\left(54 \times 4^{k+2}-27 \times 2^{k+4}+26 k+112\right) \quad 1^{9 \times 2^{k+2}-8}(B 0),
$$

and the result is still true for $k=-1$, so we have

(25) $0^{9 \times 2^{k+1}-3 k-10} 1^{3 k}(B 0) 0^{2} \quad \vdash\left(54 \times 4^{k+1}-27 \times 2^{k+3}+26 k+86\right) \quad 1^{9 \times 2^{k+1}-8}(B 0)$.

Results (25), (24) and (16) give results (b)-(d) of the theorem.

Using the rules of this theorem, we have

$$
\begin{gathered}
{ }^{\omega} 0(A 0) 0^{\omega} \vdash(1) \quad C(1) \vdash(408) \quad C(24) \vdash(14100774) \\
C(4600) \vdash(T) \quad C\left(2^{1538}-8\right) \vdash\left(2^{1538}-2\right) \quad{ }^{\omega} 01(H 1)(011)^{p} 01010^{\omega},
\end{gathered}
$$

with $T=2048 \times\left(4^{1533}-1\right) / 3-3 \times 2^{1540}+40650$ and $p=\left(2^{1538}-10\right) / 3$.

So the total time is $s\left(M_{7}\right)=2048 \times\left(4^{1533}-1\right) / 3-11 \times 2^{1538}+14141831$, and the final number of symbols 1 is $\sigma\left(M_{7}\right)=2 \times\left(2^{1538}-10\right) / 3+4$.

As in the case of Turing machine $M_{4}$, we find two ideas that explain how Turing machine $M_{7}$ works. First, machine $M_{7}$ nibbles strings of symbols 0 one by one, transforming them into strings two times longer (see transitions (14) and (15) in the proof of Theorem 9.1). Second, machine $M_{7}$ nibbles strings $(011)^{k}$ and, each time a string 011 is nibbled, the length of a string of symbols 1 is doubled (see transitions (19) and (20) in the proof of Theorem 9.1). This explains why powers of two are obtained.

Note that

$$
C(6 k+1) \vdash() \quad C(3 m) \vdash() \quad C(6 p+4) \vdash() \quad C(3 q+2) \vdash() \quad \mathrm{END},
$$


with $m=\left(2^{2 k+5}-8\right) / 3, p=3 \times 2^{m}-2, q=\left(2^{2 p+6}-10\right) / 3$. So all configurations $C(n)$ lead to a halting configuration. Those taking the most time are $C(6 k+1)$. For example, $C(7) \vdash(t)$ END with $t>10^{3.9 \times 10^{12}}$. More generally, $C(6 k+1) \vdash(t(k))$ END with $t(k)>10^{10^{10^{(3 k+2) / 5}}}$.

\section{Conclusion}

We discuss two questions as a conclusion to this article.

A. How simulating Collatz-like functions allows Turing machines to achieve high scores?

Lagarias La85] noted that the successive iterates of the $3 x+1$ function $T$ have an irregular behavior. For example, 7 iterations of function $T$ on $n=26$ lead to the value 1 , but 70 iterations are necessary on $n=27$. It seems that many Collatz-like functions have the same irregular behavior. Iterating them on small numbers may produce very long runs before stopping.

Adding parameters may increase the number of iterations by allowing the iterated values to range the set of parameters before stopping. The pure Collatz-like function with parameter $g_{3}(n, p)$ presented in Section 5 is particularly illustrative.

Another way to high scores is given by exponential Collatz-like functions such as function $g_{4}$ in Section 6. Only five iterations are performed on a blank tape, but exponential growth ensures a high score.

Irregular behavior is a condition for a Collatz-like function to be eligible to the busy beaver competition. Another condition is, of course, being computable by a very small Turing machine.

B. Are some universal devices more natural than others?

Conway [Co72] proved that there is no algorithm that, given as inputs a Collatz-like function $g$ and two integers $n, p$, outputs an answer yes or no to the question: Does there exist a positive integer $k$ such that $g^{k}(n)=p$ ? Conway [Co72, Co87] also proved that Collatz-like functions can be used to simulate all computable (also called recursive) functions. These properties can be summed up by writing that Collatz-like functions provide a universal model of computation with a m-complete decision problem.

Many universal models of computation are known: Turing machines, tag-systems, cellular automata, Diophantine equations, etc. (see [MM10]). Of course, any universal model can simulate and be simulated by any other universal model. But it is Collatz-like functions, and not another model, that appear naturally in this study. Their unexpectedly pervasive presence leads to wonder about the significance of their status among mathematical beings.

\section{ACKNOWLEGEMENT}

We thank an anonymous referee for many helpful suggestions.

\section{REFERENCES}

[Ba98] C. Baiocchi. 3n+1, UTM e Tag-systems (Italian). Dipartimento di Matematica dell'Università "La Sapienza" di Roma, 98/38, 1998.

[Ba09a] N. Bátfai. On the running time of the shortest programs. http://arxiv.org/abs/0908.1159.

[Ba09b] N. Bátfai. Recombinations of busy beaver machines. http://arxiv.org/abs/0908.4013.

[Br66] A.H. Brady. The conjectured highest scoring machines for Rado's $\Sigma(k)$ for the value $k=4$. IEEE Transactions on Electronic Computers, EC- 15: 802-803, October 1966. 
[Br83] A.H. Brady. The determination of the value of Rado's noncomputable function $\Sigma(k)$ for four-state Turing machines. Mathematics of Computation, 40 (162): 647-665, April 1983.

[Br88] A.H. Brady. The busy beaver game and the meaning of life. In: The Universal Turing machine: A Half-Century Survey, R. Herken (Ed.), Oxford University Press: 259-277, 1988.

[BM90] R.N. Buttsworth and K.R. Matthews. On some Markov matrices arising from the generalized Collatz mapping. Acta Arithmetica 55: 43-57, 1990.

[Co72] J.H. Conway. Unpredictable iterations. In: Proc. 1972 Number Theory Conference, University of Colorado, Boulder, CO: 49-52, 1972, and also in La10, 219-221.

[Co87] J.H. Conway. FRACTAN: a simple universal programming language for arithmetic. In: Open problems in Communication and Computation (T.M. Cover and B. Gopinath, Eds.), Springer: 4-26, 1987, and also in La10, 249-264.

[De84] A.K. Dewdney. Computer recreations. Scientific American 251 (5): 27, November 1984.

[GeW] G. Georgiev (Skelet). http://skelet.ludost.net/bb.

[Gr64] M.W. Green. A lower bound on Rado's sigma function for binary Turing machines. In: Proceedings of the 5th IEEE Annual Symposium on Switching Circuit Theory and Logical Design: 91-94, November 1964 .

[He09] J. Hertel. Computing the uncomputable Rado sigma function. The Mathematica Journal 11 (2): 270-283, 2009.

$[\mathrm{HuW}]$ T. Hutton. Two dimensional Turing machines. http://code.google.com/p/ruletablerepository/wiki/TwoDimensionalTuringMachines.

[Ka92] F. Kaščák. Small universal one-state linear operator algorithm. Proc. MFCS'92, Lecture Notes in Computer Science 629: 327-335, 1992.

[Ko07] S. Kohl. Wildness of iteration of certain residue-class-wise affine mappings. Advances in Applied Mathematics 39 (3): 322-328, 2007.

[LP07] G. Lafitte and C. Papazian. The fabric of small Turing machines. In: Computation and Logic in the Real World, Proceedings of the Third Conference on Computability in Europe: 219-227, June 2007.

[La85] J.C. Lagarias. The $3 x+1$ problem and its generalizations. Amer. Math. Monthly 92 (1): 3-23, 1985, and also in La10, 31-54.

[La03] J.C. Lagarias. The 3x+1 problem: an annotated bibliography (1963-1999). In [La10], 267-341, and also http://arxiv.org/abs/math/0309224.

[La06] J.C. Lagarias. The $3 x+1$ problem: an annotated bibliography (2000-). http://arxiv.org/abs/math/0608208.

[La10] J.C. Lagarias (Ed.). The Ultimate Challenge: The $3 x+1$ Problem. AMS, 2010.

[Li05] T. Ligocki. Communicated by email on 13 February 2005.

[Li06a] S. Ligocki. Communicated by email on 15 August 2006.

[Li06b] S. Ligocki. Communicated by email on 20 August 2006.

[Li10] S. Ligocki. Communicated by email on 8 May 2010.

[LR65] S. Lin and T. Rado. Computer studies of Turing machine problems. Journal of the ACM, 12 (2): 196-212, April 1965.

[Ly72] D.S. Lynn. New results for Rado's sigma function for binary Turing machines. IEEE Transactions on Computers, C-21 (8): 894-896, August 1972.

[MS90] R. Machlin and Q.F. Stout. The complex behavior of simple machines. Physica D 42: 85-98, 1990.

[Ma00] M. Margenstern. Frontier between decidability and undecidability: a survey. Theoretical Computer Science 231 (2): 217-251, 2000.

[MaW] H. Marxen. http://www.drb.insel.de/ heiner/BB/macro.html.

[MB90] H. Marxen and J. Buntrock. Attacking the Busy Beaver 5. Bulletin of the EATCS, No 40: 247-251, February 1990.

[Mi92] P. Michel. Étude de machines de Turing et complexité algorithmique. Thèse Université Paris 7, 1992.

[Mi93] P. Michel. Busy beaver competition and Collatz-like problems. Arch. Math. Logic 32 (5): 351-367, 1993.

[Mi09] P. Michel. The busy beaver competition: a historical survey. http://arxiv.org/abs/0906.3749.

[MiWa] P. Michel. Historical survey of busy beavers. http://www.logique.jussieu.fr/ michel/ha.html.

[MiWb] P. Michel. Behavior of busy beavers. http://www.logique.jussieu.fr/ michel/beh.html. 
[Mi14] P. Michel. Simulation of the Collatz $3 x+1$ function by Turing machines. http://arxiv.org/abs/1409.7322.

[MM10] P. Michel and M. Margenstern. Generalized $3 x+1$ functions and the theory of computation. In La10, 105-128.

[MuWa] R. Munafo. http://mrob.com/pub/math/ln-notes1-4.html\#mb-bb-1.

[MuWb] R. Munafo. http://mrob.com/pub/math/ln-notes1-5.html\#mb6q, and http://mrob.com/pub/math/ln-mb6q.html.

[Ra62] T. Rado. On non-computable functions. Bell Syst. Tech. J. 41 (3): 877-884, May 1962.

[Ra85] D.A. Rawsthorne. Imitation of an iteration. Mathematics Magazine 58 (3): 172-176, May 1985.

[To02] C. Tooth. Google Groups sci.math>the Turing machine known as \#r, June 28, 2002.

[Wa85] S. Wagon. The Collatz problem. The Mathematical Intelligencer 7 (1): 72-76, 1985.

[Wi98] G.J. Wirsching. The Dynamical System Generated by the $3 n+1$ Function. Lecture Notes in Mathematics 1681, Springer, 1998. 